\title{
Probing the Confining Effect of Clay Particles on an Amorphous Intercalated Dendritic Polyester
}

Kevin P. Meyers ${ }^{a}$, Jeremy J. Decker ${ }^{a}$, Brian G. Olson ${ }^{a}$, Jun Lin $^{b}$, Alexander M. Jamieson ${ }^{c}$, and Sergei Nazarenko ${ }^{a}$

Keywords: Nanocomposite, Positron annihilation lifetime spectroscopy, intercalation, hyperbranched polymer, and rigid amorphous fraction 


\section{ABSTRACT}
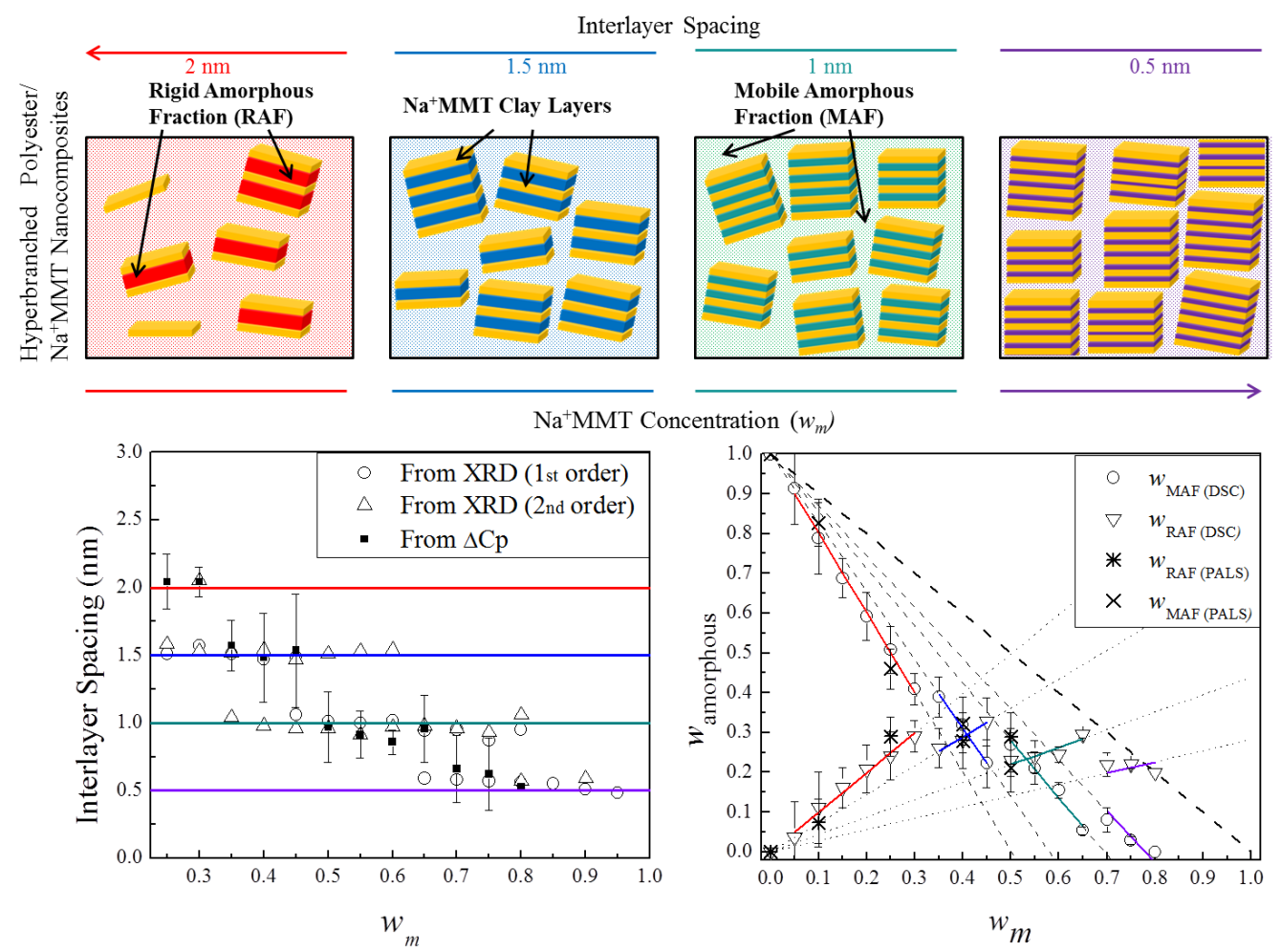

ncentration $\left(w_{m}\right)$

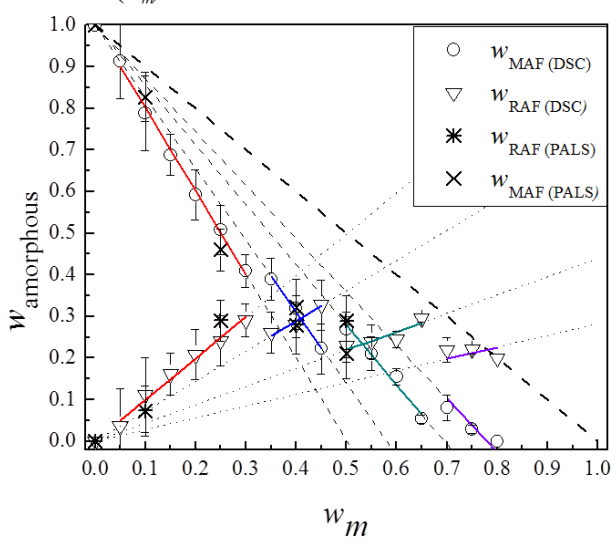

The fourth generation of a hydroxylated dendritic hyperbranched polyester (HBP) was combined with sodium montmorillonite clay $\left(\mathrm{Na}^{+} \mathrm{MMT}\right)$ using water to generate a broad range of polymer clay nanocomposites from 0 to $100 \% \mathrm{wt} / \mathrm{wt} \mathrm{Na}^{+} \mathrm{MMT}$. Analysis with differential scanning calorimetry (DSC) showed a deviation in heat capacity, $\Delta C_{p}$, with clay content at the $T_{g}$ from a two-phase trend which was attributed to the formation of an immobilized rigid amorphous fraction (RAF) in the interlayer spacing of the intercalated system. This deviation occurred in a step-like fashion which we attributed to $0.5 \mathrm{~nm}$ incremental changes in the interlayer spacing, previously observed through X-ray diffraction analysis. A simple series model was utilized to quantify these interlayer spacings based on the $\Delta C_{p}$ values and showed good correspondence with the X-ray results. The RAF was quantified from changes in heat capacity with clay content and was verified by an alternative novel positron annihilation lifetime spectroscopy (PALS) approach. The PALS quantification of the RAF was possible through an analysis of changes in the hole size thermal expansivity of the nanocomposites as a function of clay composition. Results indicated that as much as $32 \%$ by weight of the system is made up of the RAF at its maximum. 


\section{INTRODUCTION}

Polymer clay nanocomposites often exhibit chemical and physical properties that are superior to those of conventional composite materials [1-3]. The morphology of these nanocomposites can generally be classified as phase separated, intercalated, disordered intercalated, or exfoliated, depending on the interactions between the nanoclay and the polymer as well as the processing conditions [1-3]. To promote dispersion in a polymer matrix, layered silicates such as sodium montmorillonite $\left(\mathrm{Na}^{+} \mathrm{MMT}\right)$ typically require modification by surfactants to increase the organophilicity of the clay surfaces.

Various water-soluble linear polymers, such as poly(ethylene oxide) (PEO), poly(vinyl alcohol) (PVA), and poly(vinylpyrrolidinone) (PVP), have successfully been intercalated into unmodified $\mathrm{Na}^{+} \mathrm{MMT}$ clay galleries by aqueous solution casting methodologies [1-2]. However, the high viscosities of these systems can require shear intensive processing procedures, especially at high clay contents [4]. Hyperbranched polymers (HBPs) possess lower solution viscosities than linear polymers due to their more compact globular structures [5], which facilitate solution processing, even at high clay concentrations, without shear intensive procedures.

Due to their facile synthesis [6-9], dendritic hyperbranched polyester polyols (also known as Boltorn ${ }^{\mathrm{TM}}$ dendritic polyols) based on 2,2-bis-methylopropionic acid (bis-MPA) with an ethoxylated pentaerythritol core are popular model systems which preserve the essential features of dendrimers, namely high end-group functionality and a globular architecture, but possess imperfect branching and large polydispersities [5-16]. These HBPs are hydrophilic due to the presence of branch-terminal hydroxylated end groups [17], and hence are compatible with clay gallery surfaces. Experimental [18-22] and theoretical [23] studies indicate that a high interaction strength between the end functional groups of the dendritic polymers and a substrate leads to the collapse and flattening of the globular dendritic structure on the substrate.

The incorporation of polymer into clay galleries exposes numerous interfaces to the polymer matrix and the resulting polymer-substrate interactions can be probed by bulk techniques. Månson et al. [24-27] explored the structure of intercalated nanocomposite films based on surfactant-free $\mathrm{Na}^{+} \mathrm{MMT}$ clays mixed with second, third, and fourth pseudogeneration Boltorn $^{\mathrm{TM}}$ dendritic polyols. X-ray diffraction (XRD) data demonstrated that, at intermediate $\mathrm{Na}^{+} \mathrm{MMT}$ contents, the interlayer spacings of the intercalated nanocomposites correlated closely 
with estimates of the molecular diameters of the different HBP generations. It was concluded that the HBPs maintained their globular architecture in an intercalated state throughout approximately half of the compositional range. However, at the higher clay contents, the HBPs flattened within the clay galleries, leading to equivalent interlayer spacings for the $2 \mathrm{nd}$, 3rd, and 4th HPB generations.

Subsequently, we carried out a more detailed morphological analysis of the same intercalated nanocomposite system [28], specifically comprising of the 2nd and 4th pseudogenerations of Boltorn dendritic polyols and ecompassing polymer clay nanocomposite compositions from 0 to $95 \% \mathrm{wt} / \mathrm{wt} \mathrm{Na}^{+} \mathrm{MMT}$. Intercalation peaks were observed by powder XRD at and above $15 \% \mathrm{wt} / \mathrm{wt} \mathrm{Na}^{+} \mathrm{MMT}$ content for both HBP systems. In fact, intercalation was present at all clay loadings, as evidenced by TEM, but, at lower clay contents, exfoliated and disordered intercalated states were also present. The number of clay layers per intercalated stack increased with increases in $\mathrm{Na}^{+} \mathrm{MMT}$ content. The interlayer spacings for the 2nd and 4th pseudogenerations (HBP2 and HBP4) were observed to decrease in increments of approximately $0.5 \mathrm{~nm}$ as the clay content increased. Importantly, the interlayer spacings for the $2 \mathrm{nd}$ and 4 th generations of HBP were nearly identical at the same clay compositions, indicative that the interlayer spacings were independent of the HBP generation number. The interlayer spacings for both HBP2 and HBP4 decreased with increasing clay content until finally reaching a minimum spacing of $0.5 \mathrm{~nm}$ at the highest clay contents. These step-wise changes in interlayer spacings are consistent with the presence of discrete layers of flattened HBP between the clay layers. It was proposed that the HBP adsorbed onto the clay layers in solution and re-aggregated, upon solvent removal, into intercalated stacks of clay and flattened HBP. Recently, layer-by-layer intercalation of flattened Boltorn ${ }^{\mathrm{TM}}$ HBPs into $\mathrm{Na}^{+} \mathrm{MMT}$ clay galleries was confirmed by Androulaki et al. [29]. Analogous behavior was observed for $\mathrm{Na}^{+} \mathrm{MMT}$ clay based nanocomposites with a hyperbranched polyesteramide $\left(\mathrm{Hybrane}^{\mathrm{TM}}\right.$ ) and a polyamidoamine (PAMAM) dendrimer, each prepared via aqueous solution intercalation methodology [30-31]. Therefore, this trend appears to be fairly general for hydrophilic dendritic systems.

The confinement of collapsed HBP between multiple clay layers is expected to result in a sizable amount of immobilized polymer. The nature of this immobilized polymer is viewed as analogous to the concept of a rigid amorphous fraction (RAF) in semi-crystalline polymers, introduced to explain an observed discrepancy between the degree of crystallinity and the change 
in heat capacity, $\Delta C_{p}$, at the glass transition, $T_{g}$ [32]. In simplest terms, RAF represents the fraction of the amorphous phase that does not contribute to the change in $\Delta C_{p}$ at either the $T_{g}$ or $T_{m}$ (melting). It is well-established [32-44] that the RAF is due to an immobilization of the disordered polymer chains that connect the crystalline lamellae. These chains are unable to undergo long range translational motions when crystalline constraints are imposed during crystallization of the polymer melt, implying that the RAF vitrifies in the vicinity of the crystallization temperature, $T_{c}$. In contrast, the un-constrained amorphous chains, the mobile amorphous fraction (MAF), remain in the molten state at $T_{c}$ and vitrify upon cooling at the regular $T_{g}$. Complete devitrification of RAF occurs at $T_{m}$.

It is also established that the immobilized amorphous phase in nanocomposites exhibits some of the characteristics of a RAF, such as the suppression of the glass transition [45-48]. The majority of recent research on RAF at the particle interface has involved $\mathrm{SiO}_{2}$ nanocomposites that utilize $\Delta C_{p}$ analysis at the $T_{g}$ to measure the amount of RAF [49-54] and has focused on semicrystalline polymer nanocomposite systems [55-61].

Unlike crystalline phases, however, inorganic clay does not melt within the thermal stability range of the polymers. This means that, if the interactions between polymer and inorganic substrate are maintained at elevated temperatures, devitrification of the immobilized chains does not occur [45]. Indeed, this was demonstrated in a study of the dynamics of an amorphous hyperbranched polyesteramide intercalated in $\mathrm{Na}^{+} \mathrm{MMT}$ layers via quasi elasticneutron scattering [62]. It was observed that the polymer chains confined within the clay galleries exhibit dynamical behavior above the bulk $T_{g}$ similar to that of the bulk polymer below the $T_{g}$. The HBP dynamics were frozen due to the clay nanoconfinement, consistent with observed decreases in $\Delta C_{p}$ at the $T_{g}$ [62].

In the current paper we study the behavior of the immobilized RAF in surfactant-free $\mathrm{Na}^{+} \mathrm{MMT}$ clay nanocomposites generated using the 4 th generation of a Boltorn ${ }^{\mathrm{TM}}$ dendritic polyol over a very broad range of compositions $(0-100 \% \mathrm{wt} / \mathrm{wt})$. Since the $\mathrm{Na}^{+} \mathrm{MMT}$ exhibits no thermal transitions within the investigated temperature ranges, it is ideally suited to investigate polymer immobilization solely at the clay interfaces. Heat capacity measurements were used to quantify the amount of RAF as described by Wunderlich et al. for semicrystalline polymers [32]. We demonstrate that the heat capacity behavior shows a strong correlation with the earlier observed step-like behavior of interlayer spacing in these intercalated nanocomposites [28-29]. 
To probe the structure of the RAF, free volume measurements using positron annihilation lifetime spectroscopy (PALS) were employed.

PALS is a well-established, quantitative probe for free volume in polymeric materials [63-64]. In a PALS experiment, high energy positrons are injected from a radiation source into a polymer sample. The positrons thermalize via collisions with atoms and either annihilate or form a hydrogen-like pair with a secondary electron created via collision-induced ionization. In polymers, the more stable pair system, called an ortho-positronium (o-Ps), tends to localize in regions of low density, i.e. holes. Annihilation of such localized o-Ps occurs via a pickoff mechanism in which the o-Ps positron annihilates with an electron of the medium with an opposite spin. Quantitative comparisons have been established between the characteristic parameters, obtained via PALS, viz. the intensity, $I_{3}$, and lifetime, $\tau_{3}$, of the o-Ps annihilation component, and the fractional free volume, $f_{v}$, of amorphous polymers, as computed by statistical mechanical theory [65-66]. The o-Ps intensity, $I_{3}$, is typically regarded as a measure of the number density of the free volume holes. The o-Ps lifetime, $\tau_{3}$, can be related to a spherical hole radius, $R$, via the Tao-Eldrup equation, which is based on quantum mechanical and empirical arguments [67-68] as follows:

$$
\tau_{3}=0.5(n s)\left[1-\frac{R}{R_{0}}+\frac{1}{2 \pi} \sin \left(\frac{2 \pi R}{R_{0}}\right)\right]^{-1}
$$

where $R$ is the hole radius in $\AA$. $R_{0}$ equals $R+\Delta R$ where $\Delta R$ is a fitted empirical electron layer thickness of $1.66 \AA$. From $R$, the average hole free volume $v_{h}=(4 \pi / 3) R^{3}$ may be calculated. It follows that $f_{v}$ is proportional to the product $I_{3} v_{h}$.

As mineral silicate layers are too dense for o-Ps species to form, PALS is only sensitive to the HBP content of the HBP/clay nanocomposites. PALS experiments were used to assess, as a function of clay content, the average free volume hole size below and above the glass transition of the nanocomposites. The broad range of compositions prepared in this study enabled a novel opportunity to examine the free-volume behavior in intercalated polymer/clay nanocomposites. We anticipated that the RAF, which remains in the vitrified state above $T_{g}$, and the MAF, which becomes liquid at $T>T_{g}$, contribute in an additive fashion to the overall hole volume thermal expansion coefficient. Therefore, we looked forward to estimate the amount of RAF in the nanocomposites by PALS, and hence verify the DSC methodology based on the measurements of $\Delta C_{p}$ at the $T_{g}$. 


\section{EXPERIMENTAL}

\section{Materials and Sample Preparation}

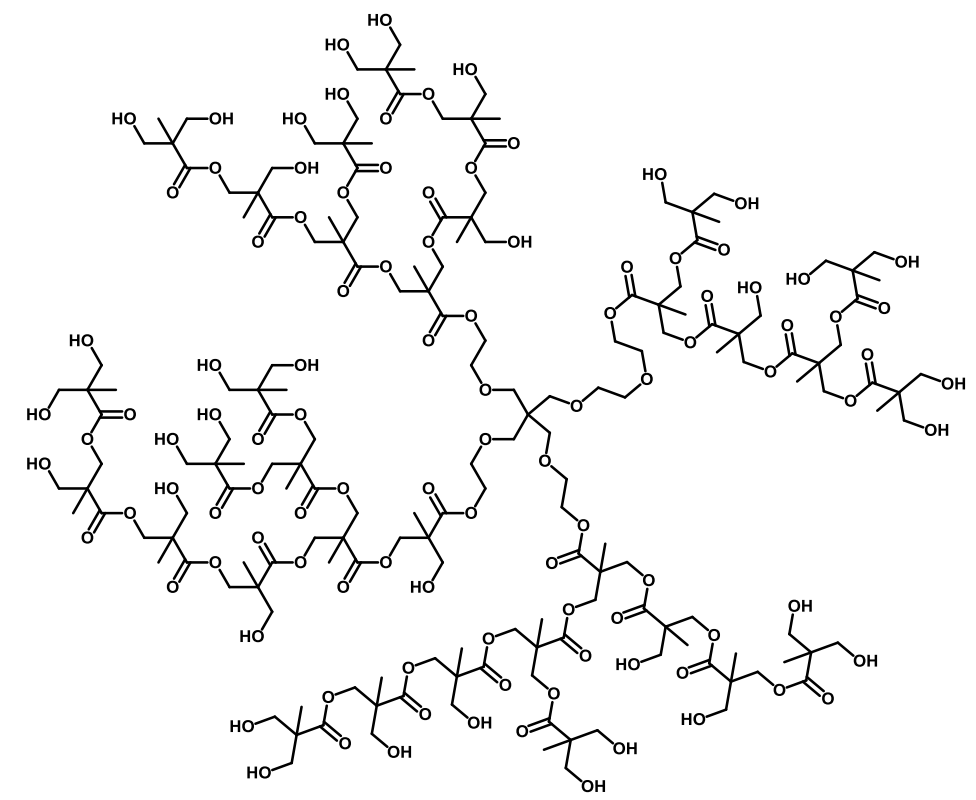

Schematic 1. Example of a fourth generation hydroxyl-functional dendritic hyperbranched polyester, Boltorn ${ }^{\mathrm{TM}}$ H40 (HBP4) accounting for imperfect branching.

Sodium montmorillonite clay $\left(\mathrm{Na}^{+} \mathrm{MMT}\right)$ Cloisite ${ }^{\circledR}$ with a cation exchange capacity (CEC) of $92.6 \mathrm{meq} / 100 \mathrm{~g}$ was purchased from Southern Clay Products. As received clay powder was sifted through a 75 micron sieve, dried at $150{ }^{\circ} \mathrm{C}$ under vacuum overnight, and stored over desiccant prior to use. The hydroxyl-functional dendritic hyperbranched polyester, Boltorn ${ }^{\mathrm{TM}}$ H40 (HBP4), was obtained from Perstorp Specialty Chemicals AB in the form of pellets. A schematic representation of the hyperbranched structure of HBP4 is shown in Schematic 1.

The nanocomposites utilized in this study were created via a solution-intercalation method and were from the same batches investigated previously [28]. The required amount of $\mathrm{Na}^{+} \mathrm{MMT}$ clay was first dispersed in deionized water at $50{ }^{\circ} \mathrm{C}$ and stirred for at least 8 hours to optimize clay delamination. The aqueous concentration of the clay was kept below $1 \% \mathrm{wt} / \mathrm{wt}$ in order to ensure that individual clay layers were well dispersed. When sufficiently diluted, $\mathrm{Na}^{+} \mathrm{MMT}$ particles are known to delaminate into single layers [69].

The required amount of Boltorn ${ }^{\mathrm{TM}}$ polyol was dissolved in boiling DI water. The concentration of polymer in water was kept at or below $10 \% \mathrm{wt} / \mathrm{wt}$ as this concentration was observed to effectively disperse and dissolve the HBP4. This solution was then quantitatively transferred into the clay dispersion. This combined solution was rapidly stirred in open air at 50 
${ }^{\circ} \mathrm{C}$ until the dispersion approached the level of the stir bar but remained in a liquid state. It was then transferred to Teflon trays and dried for two days in a convection oven at $50{ }^{\circ} \mathrm{C}$. Two further days of drying followed, under vacuum, at $120^{\circ} \mathrm{C}$. This temperature was demonstrated as optimal for removing water from Boltorn ${ }^{\mathrm{TM}}$ polyols [70]. The resulting nanocomposite films were stored over desiccant at room temperature. The mineral volume fraction of clay in the nanocomposites, $\phi_{m}$, was calculated from the corresponding mineral weight fractions, $w_{m}$, and the nanocomposite densities were measured by a buoyancy method as reported in our previous publication [28]. As also reported in our previous study, no changes in density of the amorphous HBP4 were observed in the nanocomposites [28].

\section{Characterization}

The thermal behavior of the nanocomposite and control films was evaluated using a TA Instruments Q2000 Differential Scanning Calorimeter. Heating and cooling scans were carried out at a $10{ }^{\circ} \mathrm{C} / \mathrm{min}$ rate over a range of -50 to $150{ }^{\circ} \mathrm{C}$ under a dry nitrogen atmosphere. The nanocomposites were taken directly from the desiccator and placed into sealed aluminum pans to prevent water uptake. Second heating scans were utilized for the analysis to eliminate any physical ageing effects. Second and third heating scans were self-consistent. The $T_{g}$ of the nanocomposites and the $\Delta C_{p}$, at $T_{g}$, were determined according to previously established methodology reported elsewhere [71-72]. The $T_{g}$ was taken as the point where half of the polymer was devitrified as determined from the heat capacity increase, where a line drawn median to the heat capacity lines for the glass and liquid behavior intersects the DSC curve.

The positron annihilation lifetime spectroscopy (PALS) experiments were conducted using a fast-fast coincidence system with a time resolution of $220 \mathrm{ps} .1 \times 1 \mathrm{~cm}^{2}$ area pieces were cut from each sample film for analysis. On each side of a $30 \mu \mathrm{Ci}^{22} \mathrm{Na}$ positron source, pieces of the sample films were stacked to a total thickness of $1 \mathrm{~mm}$. The sample cell was kept under vacuum during the experiments. All measurements were taken over one hour, for a total of $1 \mathrm{x}$ $10^{6}$ counts in each PALS spectrum. Temperature measurements were taken by first decreasing the temperature to $-30{ }^{\circ} \mathrm{C}$ then waiting for one hour to allow for equilibrium before beginning the first experiment. The temperature was then sequentially increased in $10^{\circ} \mathrm{C}$ steps, collecting a spectrum at each step, after waiting 10 min to allow for equilibration. The PALS spectra were tested against three and four component fits using the PATFIT 88 software package [73]. 
For transmission electron microscopy (TEM), the nanocomposite films were embedded in epoxy resin and microtomed. Approximately $90 \mathrm{~nm}$ thick sections were cut at -30 ${ }^{\circ} \mathrm{C}$ perpendicular to the film surface using a Leica cryo-ultramicrotome FC6 equipped with freshly cut glass knives. These sections were imaged using a Zeiss 109T TEM operated at $80 \mathrm{kV}$ under bright field conditions. Since the silicate layers possess a higher electron density than the surrounding HBP matrix, they appear darker in the images.

\section{RESULTS and DISCUSSION}

\section{Probing the amorphous phase of nanocomposites by DSC}

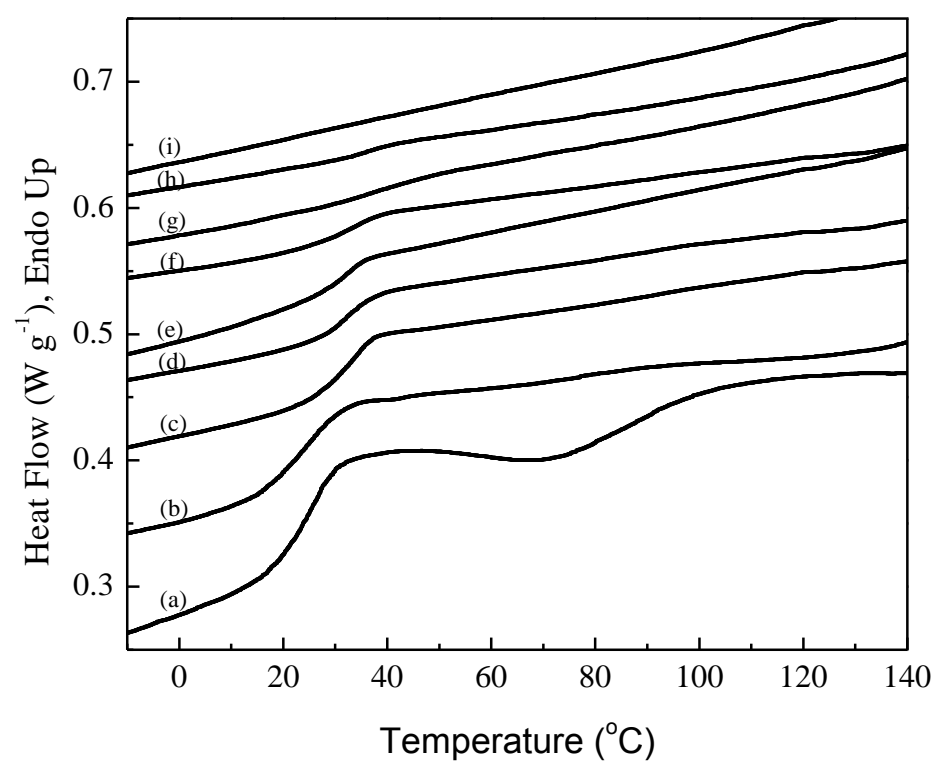

Fig. 1. DSC thermograms of HBP4/Na ${ }^{+} \mathrm{MMT}$ nanocomposites with different clay loading a) $0 \mathrm{wt} \%$, b) $10 \mathrm{wt} \%$, c) $20 \mathrm{wt} \%$, d) $30 \mathrm{wt} \%$, e) $40 \mathrm{wt} \%$, f) $50 \mathrm{wt} \%$, g) $60 \mathrm{wt} \%$, h) $70 \mathrm{wt} \%$, and i) $80 \mathrm{wt} \% \mathrm{Na}^{+} \mathrm{MMT}$. Curves vertically offset to aid the viewer.

Representative thermal scans of the nanocomposite systems and the pure HBP4 control are shown in Fig. 1. The HBP4 control exhibited a glass transition temperature at $24.2 \pm 1.2{ }^{\circ} \mathrm{C}$ followed first by exothermic and then endothermic events with corresponding minima and maxima at around $70{ }^{\circ} \mathrm{C}$ and $110{ }^{\circ} \mathrm{C}$. Žagar et al. previously attributed these exo- and endoevents to the formation and cleavage of H-bonds between multiple hydroxyl groups [9-10]. However, our most recent results, to be reported in a forthcoming publication, led us to attribute the exothermic and endothermic events, respectively, to the formation and melting of an ordered 
mesophase involving the lateral attachment of the HBP linear segments, via hydroxyl-hydroxyl or hydroxyl-carbonyl group H-bonding. The magnitude of these exo and endo events dramatically decreased with clay content until they were completely undetectable above $20 \%$ $\mathrm{wt} / \mathrm{wt} \mathrm{Na}^{+} \mathrm{MMT}$, suggesting that adding clay suppresses the formation of the mesophase, likely due to a disruption of the interchain H-bonding under confinement. Consistent with this interpretation, based on the measurements of the activation energy for the dielectric $\gamma$-relaxation, which is due to the hydroxyl group motions in similar nanocomposites, Androulaki et al. concluded [29] that the flattening of dendritic HBPs in the presence of clay surfaces impedes Hbond formation.

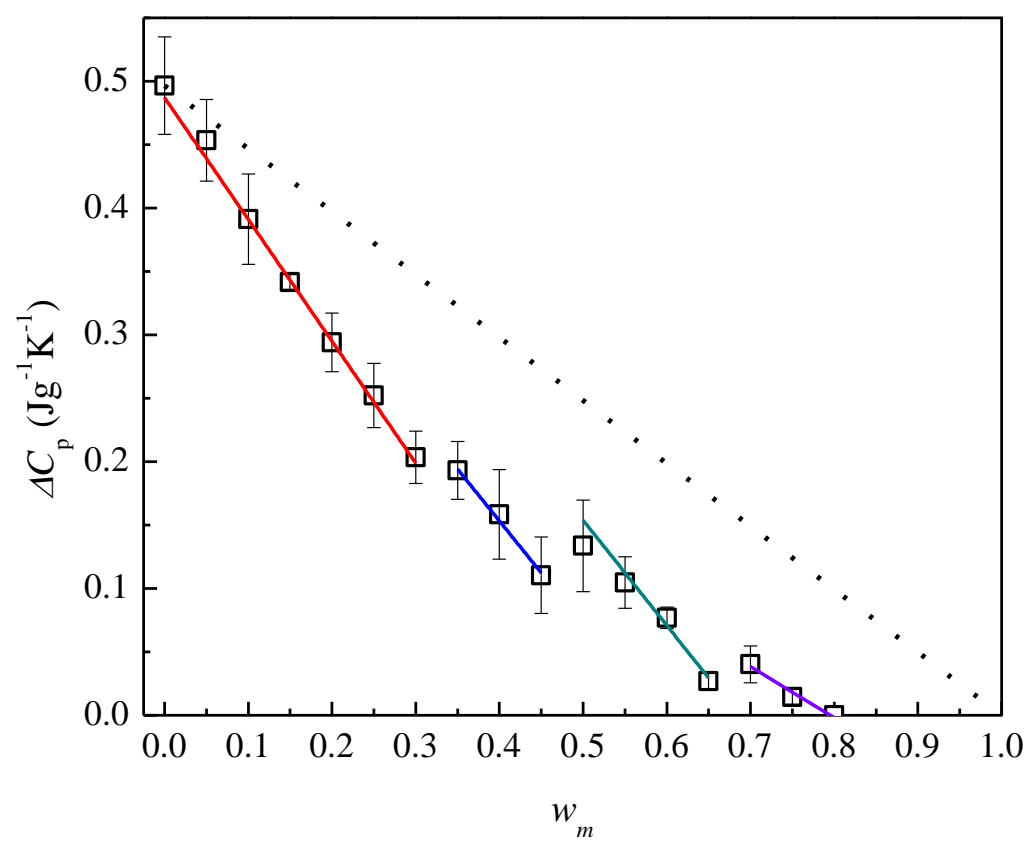

Fig. 2. $\Delta C_{p}$ at $T_{g}$ for HBP4 nanocomposites as a function of $\mathrm{Na}^{+} \mathrm{MMT}$ weight fraction, $w_{m}$. The dashed line represents the standard two-phase model prediction. Error bars represent standard deviations from multiple DSC runs. The colored shifted striations help visualize the step-wise changes in $\Delta C_{p}$.

The bulk $T_{g}$ exhibited a moderate, approximately $12{ }^{\circ} \mathrm{C}$, increase while the $\Delta C_{p}$ at $T_{g}$ decreased dramatically with the addition of clay (Fig. 1). At $80 \% \mathrm{wt} / \mathrm{wt}$ and higher clay content, the glass transition in the nanocomposites was fully suppressed. The measured $T_{g}$ and $\Delta C_{p}$ at $T_{g}$ are displayed in Table 1. Standard deviations listed are based on numerous discrete DSC measurements made on each sample. Fig. 2 depicts the heat capacity changes for the nanocomposites as a function of weight fraction of $\mathrm{Na}^{+} \mathrm{MMT}, w_{m}$. In the case of a two phase 
composite system consisting of an amorphous polymer phase and inorganic clay particles, the $\Delta C_{p}$ can be expected to decrease linearly with the clay content as depicted by the dashed line in Fig. 2. However, in reality, the $\Delta C_{p}$ behavior for the nanocomposites deviated significantly from this linear trend. The segmental mobility of a considerable portion of the amorphous HBP polymer is clearly suppressed in the presence of the clay surfaces.

The deviation from the two-phase prediction in Fig. 2 grew in significance as the clay content increased linearly to approximately $30 \% \mathrm{wt} / \mathrm{wt}$, then exhibited a series of step-like decreases, punctuated by linear increases, which can be seen in Fig. 2 as the colored shifted linear striations. These linear groupings will be described in more depth later, but are attributed to the different intercalation interlayer spacing groups as shown in Fig. 3 and depicted as colorcoordinated layers in Schematic 2. For each group, the $\Delta C_{p}$ deviates increasingly from the two phase model as more clay layers per tactoid allow for more constrained polymer. This trend follows with increasing clay content until the interlayer spacing changes (decreases) and an increase in the amount of unconstrained polymer is observed as there is less polymer immobilized between each clay layer.
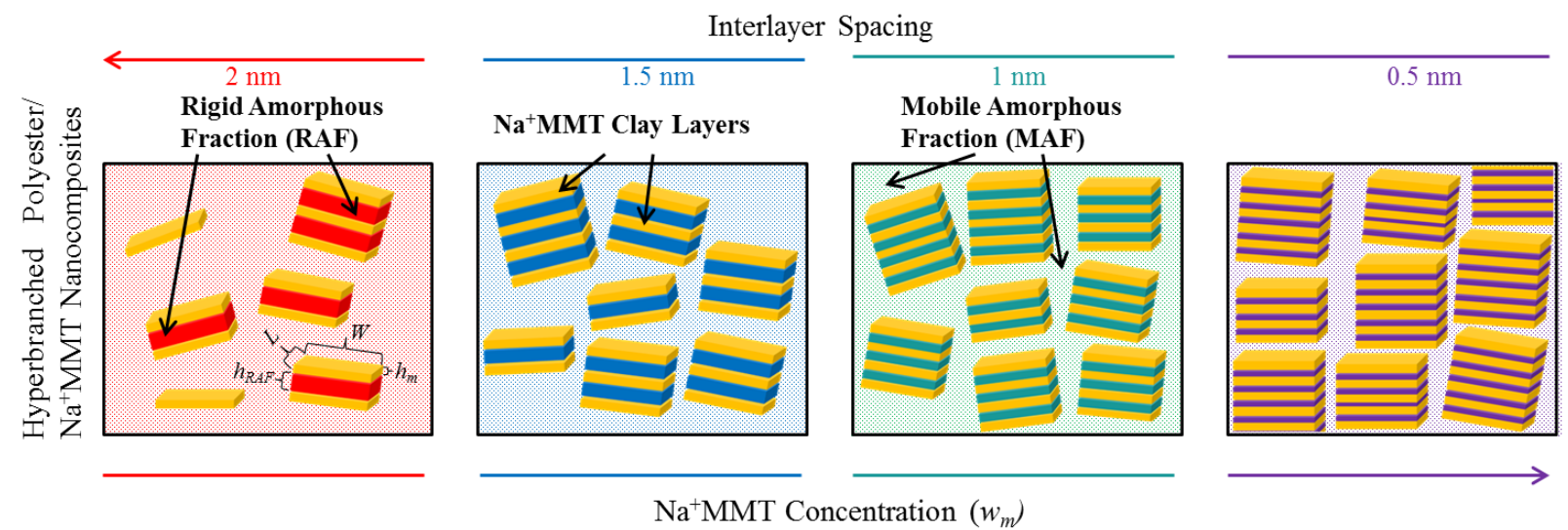

Schematic 2. Depiction of the decrease in the intercalated clay interlayer spacing with increased clay concentration. The different colors of the confined polymer phase (RAF) represent groupings of nanocomposite concentrations with distinct interlayer spacing thicknesses from 2 to $0.5 \mathrm{~nm}$. The MAF is represented by the patterned lighter shaded area surrounding the clay tactoids.

The weight fraction of the amorphous polymer phase which contributed to the step in the heat capacity at $T_{g}$, mobile amorphous fraction (MAF), was calculated based on the corresponding $\Delta C_{p}$ for the nanocomposite and $\Delta C_{p}^{o}$ for the pristine HBP4 polymer, $w_{M A F}=\frac{\Delta C_{p}}{\Delta C_{p}^{0}}$. 
The fraction of the immobilized amorphous phase, the rigid amorphous fraction (RAF), of the HPB4 polymer was calculated assuming $w_{m}+w_{R A F}+w_{M A F}=1$.

Table 1. Glass transition temperatures $\left(T_{g}\right)$, changes in heat capacity $\left(\Delta C_{p}\right)$, and calculated amorphous fractions with $\mathrm{Na}^{+} \mathrm{MMT}$ content.

\begin{tabular}{|c|c|c|c|c|c|}
\hline $\begin{array}{l}\text { Weight fraction } \\
\mathrm{Na}^{+} \mathrm{MMT} \\
\left(w_{m}\right)\end{array}$ & $\begin{array}{l}\text { Volume fraction } \\
\mathrm{Na}^{+} \mathrm{MMT} \\
\left(\varphi_{m}\right)\end{array}$ & $\begin{array}{l}\text { Glass transition } \\
\text { temperature } \\
\pm \text { std deviation } \\
\left(T_{g}\right) \\
\end{array}$ & $\begin{array}{l}\text { Heat capacity } \\
\text { jump at } T_{g} \\
\pm \text { std deviation } \\
\left(\Delta C_{p}\right)\end{array}$ & $\begin{array}{l}\text { Rigid } \\
\text { Amorphous } \\
\text { Fraction } \\
\left(w_{\mathrm{RAF}}\right) \\
\end{array}$ & $\begin{array}{l}\text { Mobile } \\
\text { Amorphous } \\
\text { Fraction } \\
\left(w_{\text {MAF }}\right) \\
\end{array}$ \\
\hline 0.00 & 0.000 & $24.2 \pm 1.2$ & $0.497 \pm 0.038$ & 0.0 & 1.0 \\
\hline 0.05 & 0.023 & $23.6 \pm 1.2$ & $0.453 \pm 0.032$ & $0.04 \pm 0.09$ & $0.91 \pm 0.09$ \\
\hline 0.10 & 0.048 & $24.7 \pm 0.5$ & $0.391 \pm 0.036$ & $0.11 \pm 0.09$ & $0.79 \pm 0.09$ \\
\hline 0.15 & 0.075 & $29.2 \pm 1.2$ & $0.342 \pm 0.006$ & $0.16 \pm 0.05$ & $0.69 \pm 0.05$ \\
\hline 0.20 & 0.102 & $31.4 \pm 1.0$ & $0.294 \pm 0.023$ & $0.21 \pm 0.06$ & $0.59 \pm 0.06$ \\
\hline 0.25 & 0.132 & $30.4 \pm 0.7$ & $0.252 \pm 0.025$ & $0.24 \pm 0.06$ & $0.51 \pm 0.06$ \\
\hline 0.30 & 0.164 & $31.7 \pm 1.2$ & $0.203 \pm 0.011$ & $0.29 \pm 0.04$ & $0.41 \pm 0.04$ \\
\hline 0.35 & 0.197 & $31.7 \pm 0.7$ & $0.193 \pm 0.023$ & $0.26 \pm 0.05$ & $0.39 \pm 0.05$ \\
\hline 0.40 & 0.233 & $30.7 \pm 0.6$ & $0.158 \pm 0.035$ & $0.28 \pm 0.07$ & $0.32 \pm 0.07$ \\
\hline 0.45 & 0.272 & $32.3 \pm 1.6$ & $0.110 \pm 0.030$ & $0.33 \pm 0.06$ & $0.22 \pm 0.06$ \\
\hline 0.50 & 0.313 & $29.2 \pm 1.4$ & $0.134 \pm 0.036$ & $0.23 \pm 0.08$ & $0.27 \pm 0.08$ \\
\hline 0.55 & 0.358 & $30.4 \pm 1.1$ & $0.105 \pm 0.020$ & $0.24 \pm 0.04$ & $0.21 \pm 0.04$ \\
\hline 0.60 & 0.406 & $37.8 \pm 2.8$ & $0.077 \pm 0.008$ & $0.25 \pm 0.02$ & $0.15 \pm 0.02$ \\
\hline 0.65 & 0.459 & $35.0 \pm 1.4$ & $0.027 \pm 0.007$ & $0.30 \pm 0.01$ & $0.05 \pm 0.01$ \\
\hline 0.70 & 0.516 & $36.0 \pm 0.8$ & $0.040 \pm 0.015$ & $0.22 \pm 0.03$ & $0.08 \pm 0.03$ \\
\hline 0.75 & 0.578 & $36.3 \pm 2.3$ & $0.014 \pm 0.006$ & $0.22 \pm 0.01$ & $0.03 \pm 0.01$ \\
\hline 0.80 & 0.646 & NA & $0.000 \pm 0.000$ & 0.20 & 0 \\
\hline
\end{tabular}

As mentioned previously, we attributed the step-like $\Delta C_{p}$ behavior (the colored striations in Fig. 2) to the step-like intercalation behavior. Naturally, a question was posed if the step-like interlayer spacing variations can be directly calculated from the $\Delta C_{p}$ behavior. The highly ordered intercalated morphology of the nanocomposites enabled us to consider a simple series model in which multiple confined polymer layers are alternated between mineral layers. 
We assume that the interlayers are composed entirely of RAF with the thickness of these layers designated as $h_{\mathrm{RAF}}$. The volume of the clay can be expressed as, $V_{m}=n L_{m} W_{m} h_{m}$, where $n$ equals the number of MMT layers and $W$ and $L$ represent the length and width of the clay stacks respectively. The thickness of the individual MMT layers, $h_{m}$, was previously determined to be $0.96 \mathrm{~nm}$. The large lateral dimensions of the clay far exceed the $1 \mathrm{~nm}$ layer thickness; therefore edge effects were neglected in this analysis. Since the RAF is assumed to be only within the MMT stacks, the volume of RAF can be expressed as $V_{R A F}=(n-1) L_{R A F} W_{R A F} h_{R A F}$. Complete surface coverage of the MMT layers by the RAF is assumed within these stacks, thus $L$ and $W$ for the RAF and MMT are assumed equal and the ratio of $V_{\mathrm{RAF}} / V_{m}$, which directly relates the heights of the RAF and MMT layers, can be expressed as:

$\frac{V_{R A F}}{V_{m}}=\frac{(n-1) h_{R A F}}{n h_{m}} \approx \frac{h_{R A F}}{h_{m}}$

TEM observations confirm that the number of layers in the intercalated stacks increases with clay content to an extent that enable us to simplify Eqn. 2 by removing the necessity to know the precise number of clay layers. Supplementary Fig. 1 shows through high and low resolution TEM micrographs that above $15 \% \mathrm{wt} / \mathrm{wt}$ the number of clay layers per stack is greater than 10 ( 15-20). This simplification will likely not hold at low clay loadings. By combining Eqn. 2 with the heat capacity prediction stated previously, $w_{M A F}=\frac{\Delta C_{p}}{\Delta C_{p}^{0}}$, and solving for $h_{\mathrm{RAF}}$, an equation was established where the interlayer spacing can be calculated from the observed $\Delta C_{p}$ values, determined by DSC, and the known clay compositions.

$h_{R A F}=\frac{\left(1-w_{m}-\Delta C_{p} / \Delta C_{p}^{0}\right) h_{m} \rho_{m}}{\rho_{R A F} w_{m}}$

As detailed in our previous publication, no changes in density of the HBP4 were observed in the nanocomposites [28]. Therefore, the densities of the MAF, $\rho_{\mathrm{MAF}}$, and the RAF, $\rho_{\mathrm{RAF}}$, were assumed to be identical with a value of $1.306 \mathrm{~g} / \mathrm{cm}^{3}$. A density, $\rho_{m}$, of $2.86 \mathrm{~g} / \mathrm{cm}^{3}$ was employed for the $\mathrm{Na}^{+} \mathrm{MMT}$ Cloisite ${ }^{\circledR}$ mineral layers in these calculations.

Fig. 3 shows the consistency between the interlayer spacings observed by XRD and those calculated from Eqn. 3. Moreover, the step-wise nature of the interlayer spacing correlates to the steps observed in Fig. 2 as marked by the linearly off-set striations for clarity. Note, the proposed DSC based analysis does not mirror the fact that different intercalated states can coexist in a given nanocomposite leading to two distinct intercalation peaks on the same diffractogram [28]. 
One plausible argument, why despite this limitation we see a good overall agreement between the interlayer spacing steps observed by XRD and predicted from Eqn. 3, is that in these mixed intercalated states one population always strongly dominates the other and is mainly reflected by DSC.

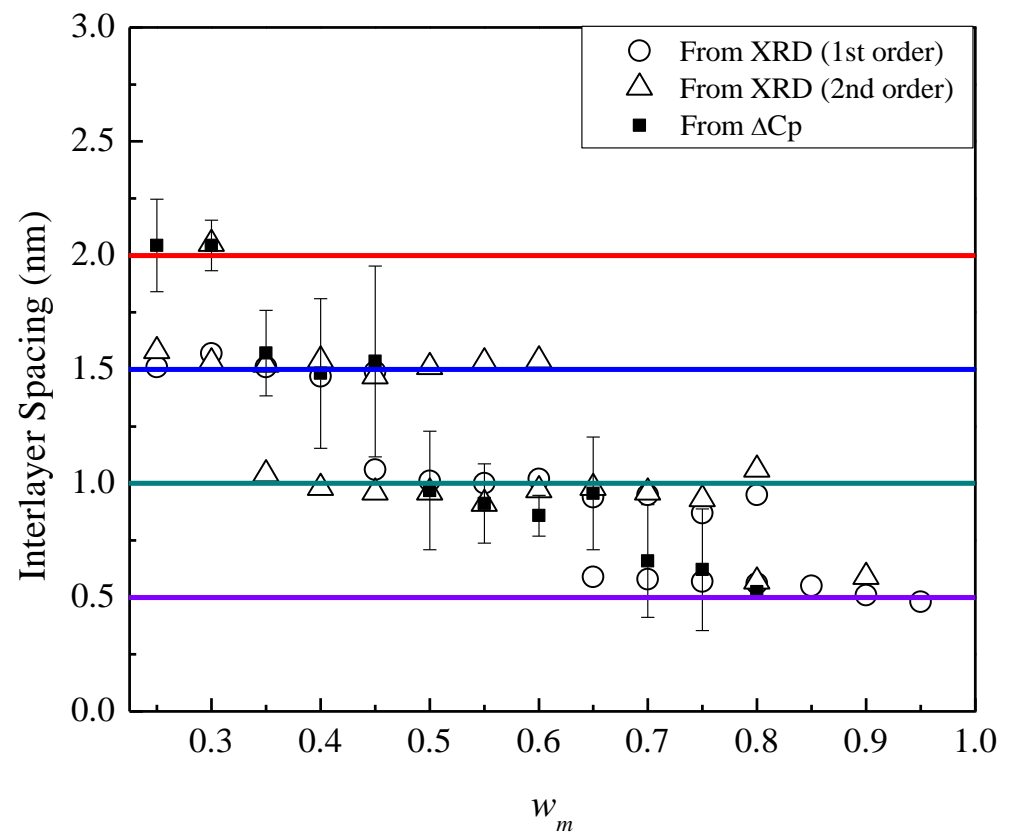

Fig. 3. Interlayer spacings predicted using Eqn. $3(\mathbb{\square})$, calculated from the experimentally determined values for $\Delta C_{p}$ at $T_{g}$. Interlayer spacings observed directly by XRD for the powdered HBP4 nanocomposites (1st order diffraction, $\bigcirc$, and 2 nd order diffraction , $\Delta$ ) are also plotted as a function of weight fraction $\mathrm{Na}^{+} \mathrm{MMT}$. Error bars represent the error propagated from the $\Delta C_{p}$ determinations. Colored gridlines represent the differentiated nanocomposite groupings with different interlayer spacings as observed in Fig. 2. 


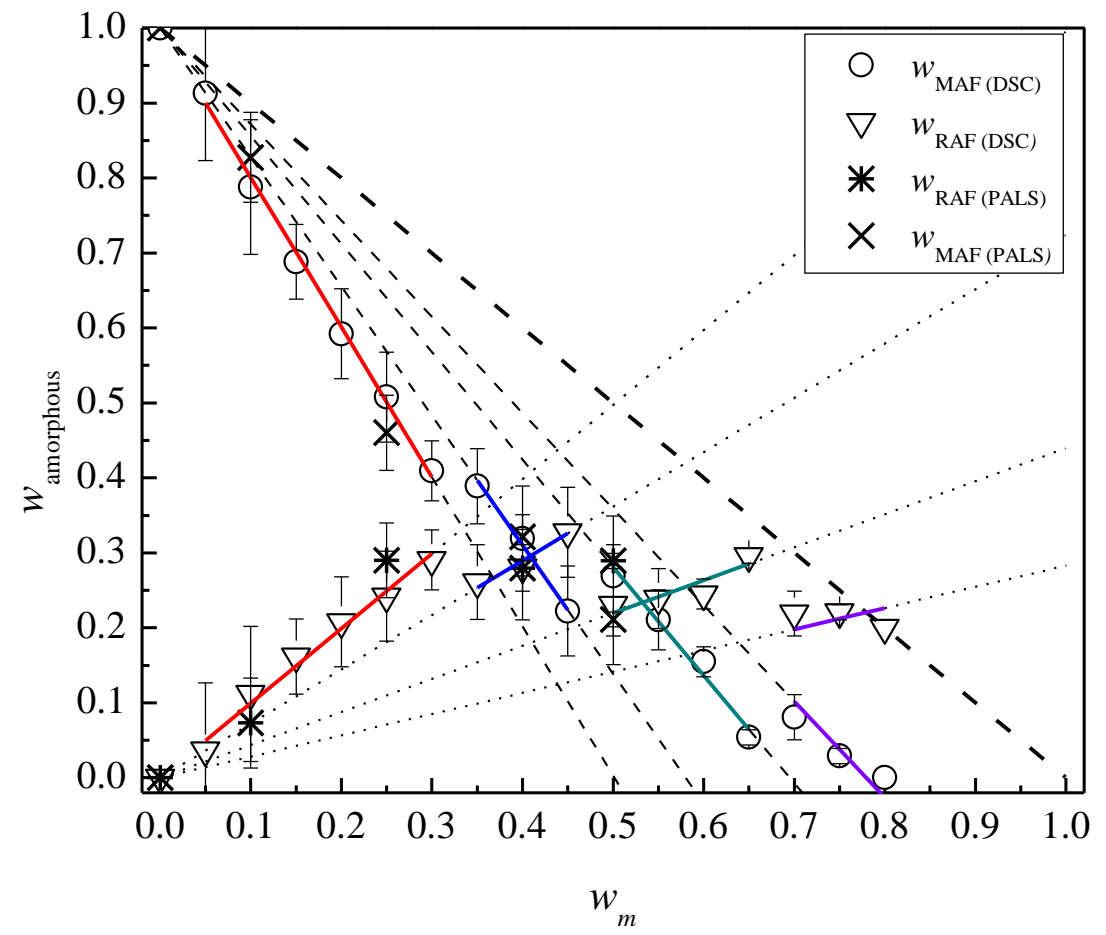

Fig. 4. Weight fractions of $\operatorname{MAF}(O)$ and $\operatorname{RAF}(\nabla)$ as determined from $\Delta C_{p}$ and weight fractions of $\operatorname{MAF}(X)$ and RAF (*) determined from PALS for HBP4 nanocomposites as a function of $w_{\mathrm{m}}$. The dashed line represents a twophase model, ie. just MAF and clay. Colored striations represent linear fits of groups of nanocomposites with increasingly smaller interlayer spacings.

As expected, the amorphous phase fractions are linearly dependent on the interlayer spacing between the platelets, $h_{\mathrm{RAF}}$. From Eqn. $3, w_{\mathrm{RAF}}$ and $w_{\mathrm{MAF}}$ are related to $h_{\mathrm{RAF}}$ by the relationship $w_{R A F}=\frac{h_{R A F} \rho_{R A F} w_{m}}{h_{m} \rho_{m}}$ and $w_{M A F}=1-w_{m}-\frac{h_{R A F} \rho_{R A F} w_{m}}{h_{m} \rho_{m}}$, respectively. The slopes of the plots of $w_{\mathrm{MAF}}$ and $w_{\mathrm{RAF}}$ versus $w_{m}$ for the previously differentiated nanocomposite concentration groups are depicted by the dotted lines. The slopes of the linear fittings of the different nanocomposite groups (labeled by different colors) clearly depict the changes in interlayer spacings with shifts from group to group. i.e., the group labeled green $(0.5$ to 0.65 $\mathrm{wt} / \mathrm{wt}$ ) has an approximately $50 \%$ reduction in its slope as compared to the group labeled red ( 0.05 to $0.3 \mathrm{wt} / \mathrm{wt})$. This correlates to the $50 \%$ reduction in interlayer spacing between the two groups, from 2 to $1 \mathrm{~nm}$ as seen in Fig. 3. Another observation is the fact that at very low clay concentrations, $<10 \% \mathrm{wt} / \mathrm{wt}$, it appears that the model still holds for this regime where the clay morphology is a mixture of exfoliated clay platelets and small tactoids with an average interlayer 
spacing of $2 \mathrm{~nm}$. This would hint that RAF can also form on the surfaces of the exfoliated clay particles ( $1 \mathrm{~nm}$ on each side), contrary to our assumption that RAF is only located in the interlayer spaces. This cannot be confirmed as many more experimental data points at these very low concentrations, with very precise $\Delta C_{p}$ measurements, would be needed before we can conclude on this issue. It is also quite possible that no RAF would be observed in a completely exfoliated system and that even at our lowest clay concentration, $0.05 \mathrm{wt} / \mathrm{wt}$, the system is still predominately intercalated. Above a $w_{m}$ of 0.8 , all of the MAF is depleted and the $w_{\mathrm{RAF}}$ would decrease in a linear two phase model manner as the system is comprised of just RAF and clay.

It is clear by comparing the changes in the RAF and MAF versus clay content trends that the RAF and MAF are dependent upon more than just the fraction of clay within the systems. The changing clay morphologies directly impact RAF formation as well.

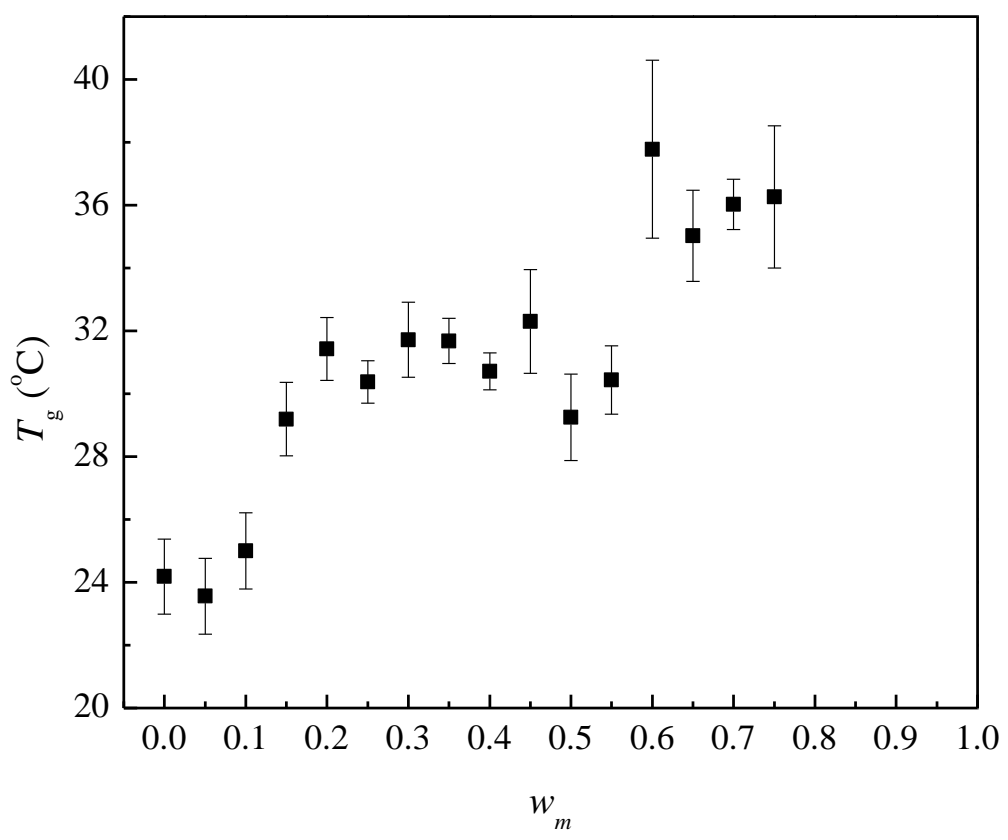

Fig. 5. Glass transition temperature as a function of weight fraction $\mathrm{Na}^{+} \mathrm{MMT}$ for $\mathrm{HBP} 4$ nanocomposites. Error bars represent standard deviations.

Finally, the changes observed by DSC in the bulk glass transition temperature with clay content of the nanocomposites are shown in Fig. 5. The $T_{g}$ remained near $24{ }^{\circ} \mathrm{C}$ at low clay concentrations until it increased between $\sim 10-30 \% \mathrm{wt} / \mathrm{wt}$ to a relative plateau region at about 31 ${ }^{\circ} \mathrm{C}$ where it remained until $\sim 50-55 \% \mathrm{wt} / \mathrm{wt}$ clay, where it increased to a final plateau at $36{ }^{\circ} \mathrm{C}$ between $60-75 \% \mathrm{wt} / \mathrm{wt}$ clay. At and above $80 \% \mathrm{wt} / \mathrm{wt} \mathrm{Na}^{+} \mathrm{MMT}, T_{g}$ detection was not 
experimentally possible due to the small amplitude of the transition peak at such low polymer concentrations (Fig. 1).

As the $T_{g}$ is assumed to be solely attributed to the MAF and not the RAF, we believe any increases in $T_{g}$ with increasing weight fraction of clay loading is a reflection of MAF that has become increasingly affected by proximity to the clay particle surfaces. Thus, we accredit the $T_{g}$ of these nanocomposites to be a combination of contributions from two MAF populations. The $T_{g}$ is affected by perturbed MAF regions, situated in the vicinity of the polymer-clay interfaces, and unperturbed MAF regions, situated at large enough distances between the clay tactoids to exhibit the original polymer $T_{g}$. The magnitude in the change in $T_{g}$ will then depend on the relative amounts of perturbed polymer and unperturbed polymer.

We believe, congruent with the data, that the $T_{g}$ behavior over the different nanoparticle concentration regimes can be explained when analyzed alongside the interlayer spacing and amorphous fraction data in Figs. 3 and 4. At very low concentrations, less than $10 \% \mathrm{wt} / \mathrm{wt}$ clay, the fraction of perturbed regions, existing only at the clay interfaces, is very small and thus the $T_{g}$ is only slightly effected by the low amount of clay inclusion. From 10 to $30 \% \mathrm{wt} / \mathrm{wt}$ clay, a dramatic increase in $T_{g}$ is observed, which can be attributed to the sharp overall decrease in MAF $(\sim 0.8$ to $\sim 0.4 \mathrm{wt} / \mathrm{wt}$, i.e. red grouping in Fig. 4$)$ taking place in this regime. As the overall MAF is reduced, the amount of perturbed MAF becomes increasingly significant with the development of more interfaces. Within the range $30-50 \% \mathrm{wt} / \mathrm{wt}$ clay, the increase in $T_{g}$ of the nanocomposite systems becomes much less pronounced. We attribute this relative constant $T_{g}$ regime, around $31^{\circ} \mathrm{C}$, to the stepwise change in MAF which decreases much less markedly, $40 \%$ to $\sim 30 \%$ $\mathrm{wt} / \mathrm{wt}$, than for the case of constant interlayer spacing. As the interlayer spacings decrease from one differentiated group to another with increased clay content, the clay tactoids incorporate more clay layers per tactoid, but with less RAF constrained in each clay layer, hence the amount of unperturbed MAF can increase. Thus, there is a trade-off in this regime that keeps the apparent $T_{g}$ relatively constant. Finally, we attribute the last regime of increased $T_{g}$, above $55 \%$ wt/wt clay, to the small concentration of MAF that remains becoming increasingly perturbed by the clay tactoids as they become closer in proximity.

The success in accurately measuring MAF and RAF content in this nanocomposite system led us to explore the use of positron annihilation lifetime spectroscopy (PALS) which we earlier applied successfully to probe free volume changes in the MAF and RAF in 
semicrystalline polyethylene terephthalate (PET) [35]. Nanocomposites should prove to be even better suited for this technique as, lacking crystallization, they are much less complex, consisting of only amorphous polymer and thermally stable nanoplatelets.

\section{Probing the amorphous fractions by PALS}

The use of PALS in the analysis of the RAF is novel with only a few attempts in semicrystalline polymers that have been reported on. Our previous work focused on using PALS to probe the free volume behavior of the RAF and MAF in a semi-crystalline PET system [35]. It was confirmed that the RAF exhibited a larger free volume in the glassy state than the MAF at ambient temperature due to the differences in their respective vitrification temperatures. The vitrification temperature for the RAF is the crystallization temperature while the MAF vitrifies at the normal glass transition temperature. This difference results in a larger excess hole free volume accumulated by the RAF upon cooling as compared to the MAF. When both fractions are melted on heating their respective free volumes merge.

To our best knowledge relatively few PALS studies have reported on the free volume in the confined amorphous phase in particulate containing nanocomposites. A few of the early attempts are reviewed in [76]. Practically all of them were limited by the extent of the mineral phase employed, polymer crystallization, and often by using surfactants, all of which made the analysis of this effect very convoluted. In contrast, the important advantages of our amorphous polymer nanocomposite system include no need of using a surfactant and the fact that these composites can be generated within a very broad range of compositions.

In the present study, we employ positron annihilation lifetime spectroscopy (PALS) in a combination with differential scanning calorimetry (DSC) to analyze the amount and nature of free volume of the RAF and MAF in the $\mathrm{Na}^{+} \mathrm{MMT} / \mathrm{HBP} 4$ nanocomposite system. The RAF content, quantified from changes in heat capacity with clay content, is compared with estimates using PALS through analysis of the glassy nature of the RAF through changes in the thermal expansivity of free volume holes as a function of clay composition.

To enable this analysis, a few research questions had to be addressed. We needed to determine if o-Ps formed inside the silicate crystals and if the o-Ps had a different probability of forming in the polymer situated in the interlayer spacings (RAF) versus the polymer between the 
clay stacks (MAF). Finally, we needed to determine if the o-Ps exhibit the same annihilation behavior in the MAF and RAF regimes.

To verify that the contribution of the o-Ps from the clay layers was indeed minimal, a PALS analysis of pure $\mathrm{Na}^{+} \mathrm{MMT}$ was performed. The clay was dried for two days under vacuum at $120{ }^{\circ} \mathrm{C}$ and then pressed in a mold to form $10 \mathrm{~mm}$ by $1 \mathrm{~mm}$ discs subjected to PALS analysis. o-Ps lifetimes in the range of 1-10 ns were not observed from PALS temperature scans of the clay.

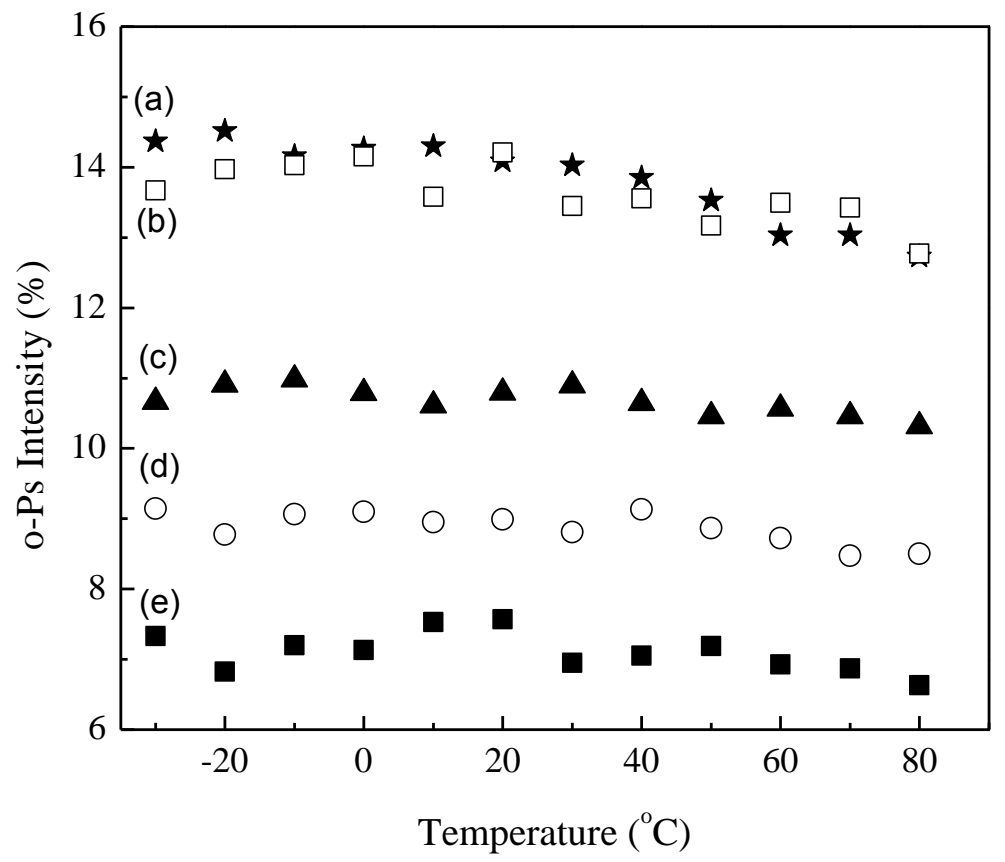

Fig. 6. Orthopositronium intensities, $I_{3}$, for $\mathrm{HBP} 4$ and $\mathrm{Na}^{+} \mathrm{MMT} / \mathrm{HBP} 4$ nanocomposites as a function of temperature at the following MMT fractions: (a) 0\%, (b) 25\%, (c) 40\%, (d) 60\% and (e) 70\% wt/wt.

In Fig. 6, we show the temperature dependence of the o-Ps intensities, $I_{3}$, for pure HBP4 and $\mathrm{Na}^{+} \mathrm{MMT} / \mathrm{HBP} 4$ nanocomposites. The o-Ps intensity, $I_{3}$, exhibited a weak temperature dependence encompassing the glass and liquid states, typical of amorphous polymers, indicating a corresponding weak temperature dependence in hole density, above and below the $T_{g}$ [77]. This also suggests that the MAF and RAF regions have consistent hole densities. If the hole densities differed, then one would expect a change in $I_{3}$ above the glass transition where only the RAF remains vitrified. Also, increased data scatter causing limited measurement reliability was experienced at high clay concentrations as the amount of polymer to analyze becomes very small, limiting the applicability of some of the data at high concentrations in further analysis. 


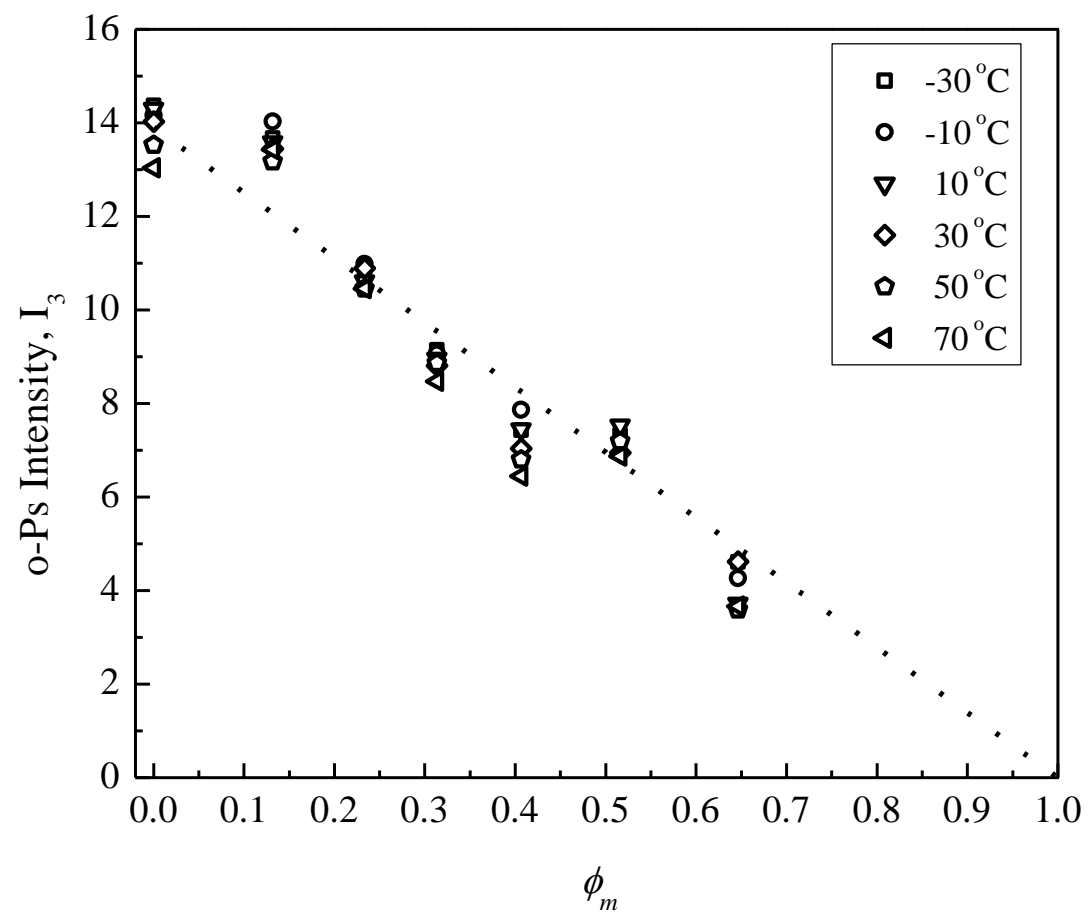

Fig. 7. o-Ps Intensity, $I_{3}$, at $-30{ }^{\circ} \mathrm{C},-10{ }^{\circ} \mathrm{C}, 10{ }^{\circ} \mathrm{C}, 30{ }^{\circ} \mathrm{C}, 50{ }^{\circ} \mathrm{C}$, and $70{ }^{\circ} \mathrm{C}$ versus volume fraction $\mathrm{Na}^{+} \mathrm{MMT}$. The dotted line represents a perfect linear correlation between $I_{3}$ and the volume fraction of clay.

The o-Ps intensity, $I_{3}$, is also plotted as a function of clay filler volume fraction in Fig. 7. The overall o-Ps intensity of the nanocomposite samples decreased linearly with increasing clay content. Similar reductions in $I_{3}$ have been observed in other clay nanocomposite systems [7880] and in certain semicrystalline systems, such as poly(ether ether ketone) where the o-Ps could not form within the polymer crystallites [81-82]. As mentioned previously, there is negligible positronium formation in the clay layers [83] limiting the regions capable of o-Ps formation to the amorphous polymer phases. Therefore, the linear trend of the o-Ps intensity with clay loading is suggestive of a two phase model dependent only on the polymer fraction and clay content. As there is no noticeable deviation from this two phase model, it can be reasoned that there is a similar probability of o-PS formation in the RAF and MAF regions. 


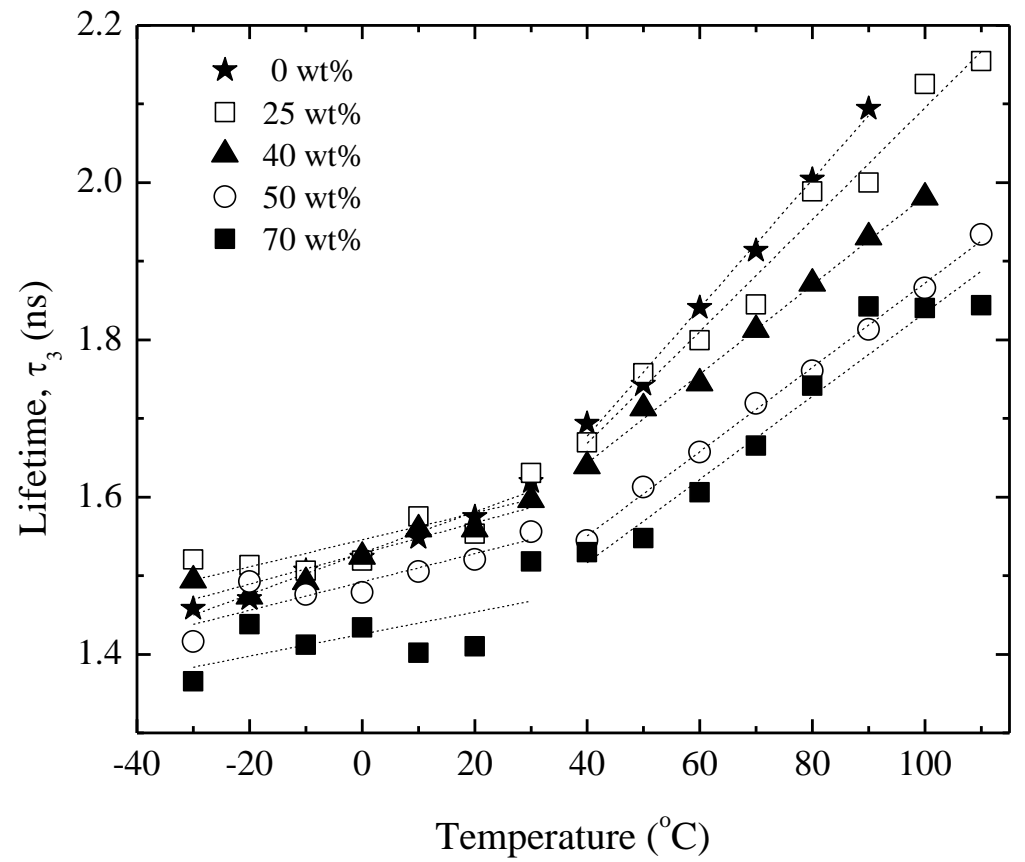

Fig. 8. Orthopositronium lifetimes, $\tau_{3}$, for HBP4 nanocomposites as a function of temperature at the following MMT fractions: $0 \%, 25 \%, 40 \%, 50 \%$ and $70 \% \mathrm{wt} / \mathrm{wt}$. The dotted lines represent linear fits of the data above and below the $T_{g}$.

As noted in Eqn. 1, the o-Ps lifetime, $\tau_{3}$, is directly related to the hole volume in the system. The temperature dependence of $\tau_{3}$ is shown in Fig. 8. The $\tau_{3}$ values below $T_{g}$ for the different clay concentrations remained very similar, as expected, except at very high loadings. While we could speculate on the cause of this decrease in $\tau_{3}$, e.g. changes in hole shape from increased orientation of the clay, as previously mentioned, data reliability is questionable at high clay concentrations where the relative amount of polymer is small. Therefore, further analysis was confined to clay concentrations at and below $50 \% \mathrm{wt} / \mathrm{wt}$. Unlike $I_{3}, \tau_{3}$ values did increase with temperature, indicative of the expansion of hole sizes on heating. The thermal expansivity of the hole volume was greater above the glass transition temperature, which is reflected in the different slopes of $\tau_{3}$ with temperature above and below $T_{g}$. The slopes of $\tau_{3}$ with temperature above $T_{g}$ decreased with increasing clay content, but the slopes below $T_{g}$ remained relatively constant, irrespective of clay content. This behavior is consistent with the results of Harms et al. in a system of poly(ethylene-alt-propylene) and hydrophobically modified silica nanoparticles [84]. 
Below $T_{g}$, both the amorphous HBP4 confined by the clay layers and the surrounding free HBP4 are in the glassy state. The constant slopes indicate that the thermal expansivity of the RAF phase is equal to that of the pure HBP4 in the glassy state. Above $T_{g}$, decreases in the $\tau_{3}$ versus $T$ slope of the nanocomposites, relative to the slope of the pure HBP4, indicate that a portion of the amorphous phase possesses a lower hole thermal expansivity than the pure HBP4 as the $\mathrm{Na}^{+} \mathrm{MMT}$ does not contribute to the o-Ps lifetimes, as established previously. Therefore, above the $T_{g}$, the free volume changes detectable by PALS can only be due to contributions from the RAF and MAF phases. Since the RAF exhibited the same thermal expansion properties as the bulk HBP4 in the glassy state, we hypothesized that the decreases in the $\tau_{3}$ slope of the nanocomposites above $T_{g}$, relative to the neat HBP4, are due to the additive contribution of the free volume hole sizes of the RAF and MAF fractions in the nanocomposites, since the RAF is expected to remain vitrified above the bulk $T_{g}$.

As the data shows that the MAF and RAF have similar o-Ps concentrations but exhibit different thermal expansivities in hole sizes above the $T_{g}$, the possibility to quantify the RAF and MAF phases became clear.

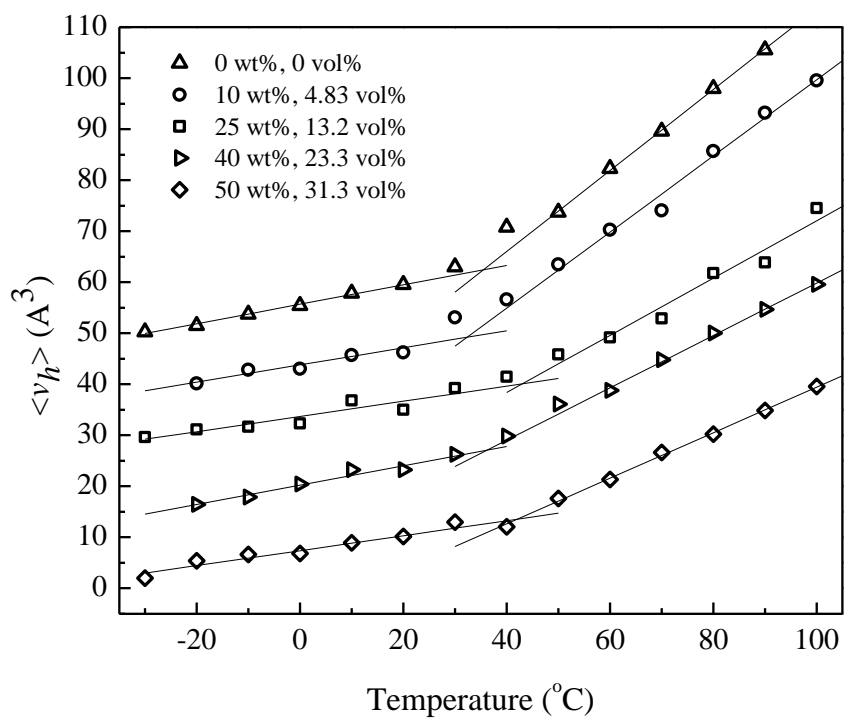

Fig. 9. Hole free volume, $v_{h}$, as a function of temperature plots for the nanocomposites. Vertically offset for clarity from $0 \% \mathrm{vol} / \mathrm{vol}$. 


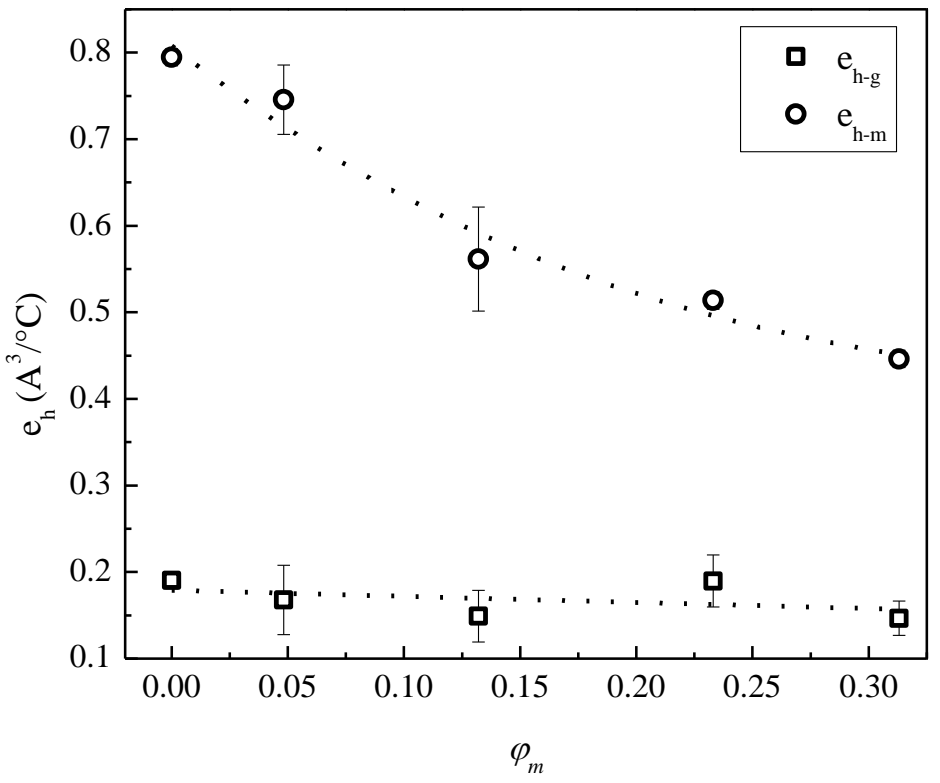

Fig. 10. Thermal expansivity of the nanocomposites systems above (o) and below ( $\square$ ) the $T_{g}$. Error bars represent the standard errors of the linear fits from Fig. 9.

The average hole size, $v_{h}$, was calculated by $v_{h}=(4 / 3) \pi R^{3}$, where $\mathrm{R}$ is calculated from Eqn. 1, and is plotted in Fig. 9. $v_{h}$ ranges from 50-120 $\AA^{3}$ over the temperature range studied. The hole thermal expansivity, $d v_{h} / d T$, of the vitrified, neat, HBP4 below $T_{g}$ was taken to be equal to that of the RAF, which remains vitrified by clay confinement above $T_{g}$. Since the contributions of RAF and MAF to $v_{h}$ are considered additive we have the following relationship $v_{h}=\frac{N^{R A F} v_{h}^{R A F}+N^{M A F} v_{h}^{M A F}}{N^{R A F}+N^{M A F}}$

where $v_{h}$ is the average hole volume as measured for the composite, $v_{h}^{\mathrm{RAF}}$ is the average hole volume as measured for RAF, and $v_{h}{ }^{\mathrm{MAF}}$ is the average hole volume as measured for MAF. $N^{\mathrm{RAF}}$ is the number of free volume holes for the RAF, and $N^{\mathrm{MAF}}$ is the number of free volume holes for the MAF. Since this analysis is dependent upon the $v_{h}$ slopes with temperature, a linear regression of $v_{h}$ in the glassy state and in the equilibrium liquid state above $T_{g}$ was required, examples of which are included in Fig. 9. For this linear regression, the data points from 20-40 ${ }^{\circ} \mathrm{C}$ are excluded as they are very close to the glass transition. The linear slope of $v_{h}$ versus $T, e_{h}$, for the nanocomposites were thusly defined as:

$e_{h}=\frac{d v_{h}}{d T}$ 
and plotted in Fig. 10 where $e_{h-g}$ and $e_{h-m}$ refer to the linear slopes below and above the $T_{g}$, respectively. Since the slope of the $v_{h}$ versus $T$ plot for all of the samples below $T_{g}$ remained constant, the linear slope of the RAF below $T_{g}, d v_{h} / d T$, was defined as $e_{h}{ }^{\mathrm{RAF}}$. As there was no HBP4 crystallinity evidenced by DSC or XRD for the pure HBP4 above $T_{g}$, the amorphous phase was considered to be composed entirely of MAF and $d v_{h} / d T$ above $T_{g}$ was defined as $e_{h}{ }^{\mathrm{MAF}}$. Based upon the negligible changes of $I_{3}$, above and below $T_{g}$, we assume that the number density of holes, $n=N_{i} / V_{i}$, is the same for RAF and MAF. Since $n$ is constant, Eqn. 4 can be rearranged as follows where $\varphi_{\mathrm{RAF}}+\varphi_{\mathrm{MAF}}+\varphi_{m}=1$.

$v_{h}=\frac{\varphi_{R A F} v_{h}^{R A F}+\varphi_{M A F} v_{h}^{M A F}}{\varphi_{R A F}+\varphi_{M A F}}$

Taking the derivative of Eqn. 6 with respect to temperature yields the final equation for the slope of $v_{h}$ with temperature, with no adjustable parameters.

$e_{h-m}=\frac{\varphi_{R A F} e_{h}^{R A F}+\varphi_{M A F} e_{h}^{M A F}}{\varphi_{R A F}+\varphi_{M A F}}$

Employing Eqn. 7 allowed for $\varphi_{\mathrm{RAF}}$ and $\varphi_{\mathrm{MAF}}$ to be extracted from the slope, $e_{h-m}$, of the $v_{h}$ plots with temperature. The slope below $T_{g}, e_{h}{ }^{\mathrm{RAF}}$, was $0.19 \AA^{3} /{ }^{\circ} \mathrm{C}$ and the slope above $T_{g}, e_{h}{ }^{\mathrm{MAF}}$, was $0.80 \AA^{3} /{ }^{\circ} \mathrm{C}$ for the pure HBP4. The correlation coefficients were at or above 0.99 for the linear regression analysis, and the results from the $v_{h}$ slope analysis are summarized in Table 2 . The resulting $\varphi_{\mathrm{RAF}}$ and $\varphi_{\mathrm{MAF}}$ determined from this analysis are shown in Table 2 and plotted in Fig. 4 relative to the loading fraction of $\mathrm{Na}^{+} \mathrm{MMT}$. The volume fractions of the amorphous phases as determined by the previous DSC analysis are included for comparative purposes in Table 2.

Table 2. Amorphous volume fractions determined from the free volume temperature coefficients of HBP4 nanocomposites.

\begin{tabular}{cccccccc}
\hline$w_{m}$ & $\varphi_{m}$ & $\begin{array}{c}e_{h-g} \\
\left(\AA^{3} /{ }^{\circ} \mathrm{C}\right)\end{array}$ & $\begin{array}{c}e_{h-m} \\
\left(\AA^{3} /{ }^{\circ} \mathrm{C}\right)\end{array}$ & $\begin{array}{c}\varphi_{\mathrm{MAF}} \\
(\mathrm{PALS})\end{array}$ & $\begin{array}{c}\varphi_{\mathrm{MAF}} \\
\left(\Delta C_{p}\right)\end{array}$ & $\begin{array}{c}\varphi_{\mathrm{RAF}} \\
(\mathrm{PALS})\end{array}$ & $\begin{array}{c}\varphi_{\mathrm{RAF}} \\
\left(\Delta C_{p}\right)\end{array}$ \\
\hline 0 & 0 & $0.19 \pm 0.01$ & $0.80 \pm 0.01$ & 1.00 & 1.00 & 0.00 & 0.00 \\
0.10 & 0.048 & $0.17 \pm 0.04$ & $0.75 \pm 0.04$ & $0.88 \pm 0.07$ & $0.83 \pm 0.10$ & $0.08 \pm 0.07$ & $0.12 \pm 0.10$ \\
0.25 & 0.132 & $0.15 \pm 0.03$ & $0.56 \pm 0.06$ & $0.53 \pm 0.06$ & $0.59 \pm 0.07$ & $0.34 \pm 0.06$ & $0.28 \pm 0.07$ \\
0.40 & 0.233 & $0.19 \pm 0.03$ & $0.51 \pm 0.01$ & $0.41 \pm 0.02$ & $0.41 \pm 0.09$ & $0.36 \pm 0.02$ & $0.36 \pm 0.09$ \\
0.50 & 0.313 & $0.15 \pm 0.02$ & $0.45 \pm 0.01$ & $0.29 \pm 0.02$ & $0.37 \pm 0.11$ & $0.37 \pm 0.02$ & $0.32 \pm 0.11$ \\
\hline
\end{tabular}


As seen in Table 2, the volume fractions of amorphous HBP4 determined from the o-Ps lifetime analysis are similar to those determined from analyzing the $\Delta C_{p}$ at $T_{g}$. This provides additional evidence that the RAF phase remains vitrified well above $T_{g}$ due to the constraints imposed by the surfaces of the montmorillonite clay layers. Therefore, it was clearly demonstrated by both the bulk thermal and nanoscopic free volume techniques that the adsorption, flattening, and confinement of the HBP lead to the vitrification of a portion of the HBP, which remains vitrified well above the bulk $T_{g}$.

\section{CONCLUSIONS}

A broad concentration range of nanocomposites, based on the fourth generation of a Boltorn $^{\mathrm{TM}}$ dendritic polyol (HBP4) combined with unmodified sodium montmorillonite clay $\left(\mathrm{Na}^{+} \mathrm{MMT}\right)$, was prepared using water as a solvent $\left(0-100 \% \mathrm{wt} / \mathrm{wt} \mathrm{Na}^{+} \mathrm{MMT}\right)$. The HBP4 exhibits a DSC glass transition temperature $\sim 24{ }^{\circ} \mathrm{C}$, followed by exothermic and endothermic events, attributed to the formation and melting of an ordered mesophase, whose formation is suppressed on addition of clay to form a nanocomposite.

With the addition of clay, the bulk $T_{g}$ exhibited a modest increase, while the $\Delta C_{p}$ at $T_{g}$ showed a substantial decrease. The $\Delta C_{p}$ behavior for the nanocomposites deviated significantly from the linear trend expected for a two-phase composite in an unusual step-like fashion indicative of suppression of the segmental mobility of the amorphous HBP polymer. At and above $80 \% \mathrm{wt} / \mathrm{wt}$ of clay, the glass transition in the nanocomposites was fully suppressed. The step-like deviation from the two-phase prediction was demonstrated to correlate to step-like changes in intercalation interlayer spacing groupings observed in a previous study [28]. The weight fraction of the MAF ( $w_{\mathrm{MAF}}$ ), which contributed to the step in the heat capacity at $T_{g}$ was calculated based on the corresponding $\Delta C_{p}$. A simple series mathematical model in which multiple confined polymer layers alternate between mineral layers enabled calculation of interlayer spacings from the experimental $\Delta C_{p}$ data which were in excellent agreement with those determined by XRD.

From knowledge of $w_{\mathrm{MAF}}$ for each clay content we were able to calculate the amount of $\mathrm{RAF}\left(\right.$ since $w_{m}+w_{\mathrm{RAF}}+w_{\mathrm{MAF}}=1$ ). As much as $\sim 32 \% \mathrm{wt} / \mathrm{wt}$ of the system was made up of RAF at its maximum. Incremental steps in interlayer spacings observed at 0.3-0.35, 0.45-0.5, and 
0.65-0.7 wt/wt clay correlated to abrupt increases in $w_{\mathrm{MAF}}$ (decrease in $w_{\mathrm{RAF}}$ ) at each step, followed by a linear decrease in $w_{\mathrm{MAF}}$ (increase in $w_{\mathrm{RAF}}$ ) with increasing clay content until the next step is reached. Clearly, the changes in the RAF and MAF content depend not only on the fraction of clay within the system but also on the changes in clay morphology.

As the $T_{g}$ is attributed solely to the MAF and not the immobilized RAF, we believe the observed increases in $T_{g}$ with increasing weight fraction of clay loading are a reflection of a MAF fraction whose mobility has become perturbed by the proximity to the clay particles. Thus, we attribute the $T_{g}$ of these nanocomposites to be a result of the combination of two distinct MAF populations, perturbed and unperturbed MAF situated at large enough distances between the clay tactoids to exhibit the bulk polymer $T_{g}$. The magnitude of the change in $T_{g}$ then depends on the relative amounts of each population.

Positron annihilation spectroscopy (PALS) was used to gain information on the temperature dependence of free volume hole density and average hole sizes from $I_{3}$ and $\tau_{3}$, respectively encompassing the glassy and liquid states. The weak temperature dependence of $I_{3}$ indicated similar o-Ps formation probabilities in RAF and MAF. This enabled a novel nanocopic approach to estimate $w_{\mathrm{RAF}}$ and $w_{\mathrm{MAF}}$ via PALS from the thermal expansivities of hole sizes in the liquid and glassy states. The volume fractions of RAF and MAF of the HBP4 nanocomposites determined from o-Ps lifetime analysis proved to be consistent with those determined from analysis of $\Delta C_{p}$ at $T_{g}$. This serves to verify the glassy nature of the RAF constrained by the montmorillonite layers at elevated temperatures.

\section{Acknowledgements}

The authors are grateful to the National Science Foundation for support of this work through the Center for Layered Polymeric Systems (DMR 0423914) and MRSEC (DMR 0213883). 


\section{References}

[1] Alexandre M, Dubois P. Polymer-layered silicate nanocomposites: preparation, properties, and uses of a new class of materials. Mater. Sci. Eng. 2000; $28: 1$.

[2] Ray SS, Okamoto M. Polymer/layered silicate nanocomposites: a review from preparation to processing. Prog. Polym. Sci. 2003; 28: 1539.

[3] Tjong SC. Structural and mechanical properties of polymer nanocomposites. Mater. Sci. Eng. 2006; 53: 73.

[4] Strawhecker KE, Manias E. Structure and Properties of Poly(vinyl alcohol)/Na Montmorillonite Nanocomposites. Chem. Mater. 2000; 12: 2943.

[5] Jikei M, Kamimoto M. Hyperbranched polymers: a promising new class of materials. Prog. Polym. Sci. 2001; 26: 1233.

[6] Zhu PW, Zheng S, Simon G. Dielectric Relaxations in a Hyperbranched Polyester with Terminal Hydroxyl Groups: Effects of Generation Number. Macromol. Chem. Phys. 2001; 202: 3008.

[7] Luciani A, Plummer CJG, Nguyen, T, Garamszegi L, Manson JE. Rheological and Physical Properties of Aliphatic Hyperbranched Polyesters. J. Polym. Sci. Part B: Polym. Phys. 2004; 42: 1218.

[8] Žagar E, Žigon M. Characterization of a Commercial Hyperbranched Aliphatic Polyester Based on 2,2-Bis(methylol)propionic Acid. Macromolecules 2002; 35: 9913.

[9] Žagar E, Huskić M, Žigon M. Macrom. Structure-to-Properties Relationship of Aliphatic Hyperbranched Polyesters. Chem. Phys. 2007; 208: 1379.

[10] Žagar E, Huskić M, Grdadolnik J, Žigon M, Zupanćić-Valant A. Effect of Annealing on the Rheological and Thermal Properties of Aliphatic Hyperbranched Polyester Based on 2,2-Bis(methylol)propionic Acid. Macromolecules 2005; 38: 3933.

[11] Žagar E, Žigon M, Podzimek S. Characterization of Commercial Aliphatic Hyperbranched Polyesters. Polymer 2006; 47: 166.

[12] Tanis I, Karatasos K. Local Dynamics and Hydrogen Bonding in Hyperbranched Aliphatic Polyesters. Macromolecules 2009; 42: 9581.

[13] Malmström E, Johansson M, Hult A. Hyperbranched Aliphatic Polyesters. Macromolecules 1995; 28: 1698. 
[14] Burgath A, Sunder A, Frey H. Role of Cyclization in the Synthesis of Hyperbranched Aliphatic Polyesters. Macromol. Chem. Phys. 2000; 201: 782.

[15] Malmström E, Hult A. Kinetics of Formation of Hyperbranched Polyesters Based on 2,2bis(methylol)propionic Acid. Macromolecules 1996; 29: 1222.

[16] Magnusson H, Malmström E, Hult A. Structure Buildup in Hyperbranched Polymers from 2,2-Bis(hydroxymethyl)propionic Acid. Macromolecules 2000; 33: 3099.

[17] Zeiner T, Shrader P, Enders S, Browarzik DJ. Phase- and Interfacial Behavior of Hyperbranched Polymer Solutions. Fluid Phase Equilib. 2011; 302: 321.

[18] Tsukruk VV. Dendritic Macromolecules at Interfaces. Adv. Mater. 1998; 10(3): 253.

[19] Peleshanko S, Tsukruk VV. The architectures and surface behavior of highly branched molecules. Prog. Polym. Sci. 2008; 33: 523.

[20] Tsukruk VV, Rinderspacher F, Bliznyuk VN. Self-Assembled Multilayer Films from Dendrimers. Langmuir 1997; 13: 2171.

[21] Bakshi MS, Sood R, Kaur G, Sakai K, Yoshimura T, Esumi K. Characterization of aliphatic and aromatic polyester hyperbranched dendrimers by AFM imaging. Colloid Polym. Sci. 2005; 284: 74.

[22] Sheiko SS, Musafarov AM, Winkler RG, Getmanova EV, Eckert G, Reineker P. Contact Angle Microscopy on a Carbosilane Dendrimer with Hydroxyl End Groups: Method for Mesoscopic Characterization of the Surface Structure. Langmuir 1997; 13: 4172.

[23] Mansfield ML. Surface adsorption of model dendrimers. Polymer 1996; 37(17): 3835.

[24] Plummer CJG, Gramszegi L, Leterrier Y, Rodlert M, Månson JE. Hyperbranched Polymer Layered Silicate Nanocomposites. Chem. Mater. 2002; 14: 486.

[25] Rodlert M, Plummer CJG, Grünbauer HJM, Månson JE. Hyperbranched Polymer/Clay Nanocomposites. Adv. Eng. Mater. 2004; 6(9): 715.

[26] Rodlert M, Plummer CJG, Gramszegi L, Leterrier Y, Grunbauer HJM, Månson JE. Hyperbranched Polymer/Montmorillonite Clay Nanocomposites. Polymer 2004; 45: 949.

[27] Rodlert M, Plummer CJG, Leterrier Y, Månson JE. Rheological Behavior of Hyperbranched Polymer/Montmorillonite Clay Nanocomposites J. Rheol. 2004; 48(5): 1049. 
[28] Decker JJ, Chvalun SN, Nazarenko S. Intercalation behavior of hydroxylated dendritic polyesters in polymer clay nanocomposites prepared from aqueous solution. Polymer 2011; 52: 3943.

[29] Androulaki K, Chrissopoulou K, Prevosto D, Labardi M, and Anastasiadis SH. Dynamics of Hyperbranched Polymers Under Confinement: A Dielectric Relaxation Study. ACS Appl. Mater. Interfaces 2015; 7: 12387

[30] Fotiadou S, Karageorgaki C, Chrissopoulou K, Karatasos K, Tanis I, Tragoudaras D, Frick B, Anastasiadis SH. Structure and Dynamics of Hyperbranched Polymer/Layered Silicate Nanocomposites. Macromolecules 2013; 46: 2842.

[31] Liyanage AU, Ikhuoria EU, Adenuga AA, Remcho VT, Lerner MM. Synthesis and Characterization of Low-Generation Polyamidoamine (PAMAM) Dendrimer-Sodium Montmorillonite (Na-MMT) Clay Nanocomposites. Inorg. Chem. 2013; 52: 4603.

[32] Wunderlich B. Reversible crystallization and the rigid-amorphous phase in semicrystalline macromolecules. Prog. Polym. Sci. 2003; 28: 383.

[33] Wunderlich B. Calorimetry of Nanophases of Macromolecules. Int. J. Thermophys. 2007; 28: 958.

[34] Lin J, Shenogin S, Nazarenko S. Oxygen Solubility and Specific Volume of Rigid Amorphous Fraction in Semicrystalline Poly(ethylene terephthalate). Polymer 2002; 43: 4733.

[35] Olson BG, Lin J, Nazarenko S, Jamieson AM. Positron Annihilation Lifetime Spectroscopy of Poly(ethylene terephthalate): Contributions from Rigid and Mobile Amorphous Fractions. Macromolecules 2003; 36: 7618.

[36] Righetti MC, Tombari E, Di Lorenzo ML. The Role of the Crystallization Temperature on the Nanophase Structure Evolution of Poly[(R)-3-hydroxybutyrate]. J. Phys. Chem. B 2013; 117: 12303.

[37] Di Lorenzo ML, Androsch R, and Stolte I. Tailoring the rigid amorphous fraction of isotactic polybutene-1 by ethylene chain defects. Polymer 2014; 55: 6132.

[38] Hamonic F, Miri V, Saiter A, and Dargent E. Rigid amorphous fraction versus oriented amorphous fraction in uniaxially drawn polyesters. Eur. Polym. J. 2014; 58: 233.

[39] Beckingham BS, Ho V, and Segalman RA. Formation of a Rigid Amorphous Fraction in Poly(3-(2'-ethyl) hexylthiophene). ACS Macro Lett. 2014; 3: 684. 
[40] Righetti MC, Laus M, and Di Lorenzo ML. Temperature dependence of the rigid amorphous fraction in poly(ethylene terephthalate). Eur. Polym. J. 2014; 58: 60.

[41] Righetti MC, Laus M, Di Lorenzo ML. Rigid amorphous fraction and melting behavior of poly(ethylene terephthalate). Colloid Polym. Sci. 2014; 292: 1365.

[42] Tognana S and Salgueiro W. Influence of the rigid amorphous fraction and segregation during crystallization in PHB/DGEBA blends. Polym. J. 2015: 1.

[43] Massa CA, Pizzanelli S, Bercu V, Pardi L, and Leporini D. Constrained and Heterogeneous Dynamics in the Mobile and the Rigid Ammorphous Fractions of Poly(dimethylsiloxane): A Multifrequency High-Field Electron Paramagnetic Resonance Study. Macromolecules 2014; 47, 6748.

[44] Remy R, Wei S, Campos LM, and Mackay ME. Three-Phase Morphology of Semicrystalline Polymer Semiconductors: A Quantitative Analysis. ACS Macro Lett. 2015; 4: 1051.

[45] Sargsyan A, Tonoyan A, Davtyan S, Schick C. The amount of immobilized polymer in PMMA $\mathrm{SiO}_{2}$ nanocomposites determined from calorimetric data. Eur. Polym. J. 2007; 43: 3113.

[46] Corcione EC, Maffezzoli A. Glass transition in thermosetting clay-nanocomposite polyurethanes. Thermochim Acta 2009; 485: 43.

[47] Wurm A, Ismail M, Kretzschmar B, Pospiech D, Schick C. Retarded Crystallization in Polyamide/Layered Silicates Nanocomposites Caused by an Immobilized Interphase. Macromolecules 2010; 43: 1480.

[48] Fotiadou S, Chrissopoulou K, Frick B, Anastasiadis SH. Structure and Dynamics of Polymer Chains in Hydrophilic Nanocomposites. J. Polym. Sci., Part B: Polym. Phys. 2010; 48: 1658 .

[49] Sari MG, Shahbazi M, Pakdel AS. Developing a Novel Hyperbranched PolymerModified PP/Clay Nanocomposite: Characteristics Investigation. Polymer-Plastics Technology and Engineering 2014; 53: 1561

[50] Sargsyan A, Tonoyan A, Davtyan S, Schick C. The amount of immobilized polymer in PMMA $\mathrm{SiO}_{2}$ nanocomposites determined from calorimetric data. Eur. Polym. J. 2007; 43: 3113 . 
[51] Sargsyan A, Tonoyan A, Davtyan S, and Schick C. Rigid Amorphous Fraction in Polymer Nano-Composites. NATAS Notes 2007; 39: 6.

[52] Pissis P, Klonos P, and Kyritsis A. Interfacial Effects in Polymer Nanocomposites Studied by Dielectric and Thermal Techniques. IEEE 2011; 67.

[53] Davtyan SP, Tonoyan AO, Varderesyan AZ, Muller SC. Frontal compolymerization in the presence of nano-particles. Eur. Polym. J. 2014; 57: 182.

[54] Kim S, Nguyen NA, Wie JJ, and Park HS. Manipulating the glass transition behavior of sulfonated polystyrene by functionalized nanoparticle inclusion. Nanoscale 2015; 7: 8864.

[55] Chrissopoulou K, Fotiadou S, Frick B, and Anastasiadis SH. Structure and Dyanamics in Hydrophillic Polymer/ Layered Silicate Nanocomposites. Macromol. Symp. 2013; 331332: 50 .

[56] Signori F, Pelagaggi M, Bronco S, and Righetti MC. Amorphous/crystal and polymer/filler interphases in biocomposites from poly(butylene succinate). Thermochim Acta 2012; 543: 74.

[57] Karami S and Lafleur PG. Role of Chain Dynamics and Topological Confinements in Cold Crystallization of PLA-Clay Nanocomposites. Polym. Eng. Sci. 2015; 55: 1310

[58] Klonos P, Kripotou S, Kyritsis A, Papageorgiou GZ, Bikiaris D, Gournis D, and Pissis P. Glass transistion and segmental dyanmics in poly(L-lactic acid)/ graphene oxide nanocomposites. Thermochim Acta 2015; 617: 44.

[59] Charlon S, Marais S, Dargent E, Soulestin J, Sclavons M, and Follain N. Structure-barrier property relationship of biodegradable poly(butylene succinate) and poly[(butylene succinate)-co-(butylene adipate)] nanocomposites: influence of the rigid amorphous fraction. Phys. Chem. Chem. Phys. 2015; 17: 29918.

[60] Rath SK, Sudarshan K, Bhavsar RS, Kharul UK, Pujari PK, Patri M, and Khakhar DV. Characterizing the nanoclay induced constrained amorphous region in model segmented polyurethane-urea/clay nanocomposites and its implications on gas barrier properties. Phys. Chem. Chem. Phys. 2016; 18: 1487.

[61] Purohit PJ, Wang D, Wurm A, Schick C, and Schonhals A. Eur. Polym. J. 2014; 55: 48. 
[62] Fotiadou S, Karageorgaki C, Chrissopoulou K, Karatasos K, Tanis I, Tragoudaras D, Frick B, Anastasiadis SH. Structure and Dynamics of Hyperbranched Polymer/Layered Silicate Nanocomposites. Macromolecules 2013; 46: 2842.

[63] Pethrick RA. Positron annihilation-A probe for nanoscale voids and free volume? Prog. Polym. Sci. 1997; 22: 1.

[64] Jean YC, Mallon PE, Schrader DM (Eds). Principles and Applications of Positron and Positronium Chemistry. River Edge, NJ: World Scientific; 2003.

[65] Higuchi H, Yu Z, Jamieson AM, Simha R, McGervey JD. Thermal history and temperature dependence of viscoelastic properties of polymer glasses: relation to free volume quantities. J. Polym. Sci. Polym. Phys. 1995; 33(17): 2295.

[66] Simha R, Somcynsky T. On the Statistical Thermodynamics of Spherical and Chain Molecule Fluids. Macromolecules 1969; 2: 342.

[67] Eldrup M, Lightbody D, Sherwood NJ. The temperature dependence of positron lifetimes in solid pivalic acid. Chem. Phys. 1981; 63: 51.

[68] Tao SJ. Positronium annihilation in molecular substances. J. Chem. Phys. 1972; 56: 5499.

[69] Lagaly G, Ziesmer S. Colloid chemistry of clay minerals: the coagulation of montmorillonite dispersions. Adv. Colloid Interface Sci. 2002; 100: 105.

[70] Carmezini G, Mackay ME, Sauer BB, Kampert W. Manipulation of Hyperbranched Polymers' Conformation. Chem. Mater. 2002; 14: 819.

[71] Cheng SZD, Cao M-Y, Wunderlich B. Glass transition and melting behavior of poly(oxy1,4-phenyleneoxy-1,4-phenylenecarbonyl-1,4-phenylene) (PEEK). Macromolecules 1986; 19: 1868.

[72] Richardson MJ. In: Mathot VBF (Eds.) Calorimetry and thermal analysis of polymers. Munich: Hanser; 1993. 170.

[73] Kirkegard P, Eldrup M, Morgesen OE, Pedersen, NJ. Program system for analysing positron lifetime spectra and angular correlation curves. Comput. Phys. Commun. 1981; 23: 307.

[74] Grebowicz J, Lau SF, Wunderlich BJ. The thermal properties of polypropylene. J. Polym. Sci. Polym. Symp. 1984; 71: 19.

[75] Suzuki H, Grebowicz J, Wunderlich BJ. Heat capacity of semicrystalline, linear poly(oxymethylene) and poly(oxyethylene). Macromol. Chem. 1985; 186: 1109. 
[76] Jamieson AM, Olson BG, Nazarenko S. Positron Annihilation Lifetime Studies of Free Volume in Heterogeneous Polymer Systems In L. Utrackie and A. Jamieson (Eds.), Polymer Physics: From Suspensions to Nanocomposites and Beyond. Hoboken, NJ: John Wiley \& Sons, Inc.; 2010. 473.

[77] Yu Z, Yahsi U, McGervey JD, Jamieson AM, Simha R. Molecular Weight-Dependance of Free Volume in Polystyrene Studied by Positron Annihilation Measurements. J. Polym. Sci. Pol. Phys. 1994; 32: 2637.

[78] Choudalakis G, Gotsis AD. Free volume and mass transport in polymer nanocomposites. Curr. Opin. Colloid Interface Sci. 2012; 17: 132.

[79] Wang Y-Q, Wu, Y-P, Zhange H-F, Zhang L-Q, Wang B, Wang Z-F. Free Volume of Montmorillonite/Styrene-Butadiene Rubber Nanocomposites Estimated by Positron Annihilation Lifetime Spectroscopy. Macromol. Rapid Commun. 2004; 25: 1973.

[80] Wang ZF, Wang B, Qi N, Zhang HF, Zhang LQ. Influence of Fillers on Free Volume and Gas Barrier Properties in Styrene-butadiene Rubber Studied by Positrons. Polymer 2005; 46: 719.

[81] Nakanishi H, Jean YC. Dynamics of Excess Free Volume in Semicrystalline PEEK Studied by Positron Annihilation. Macromolecules 1991; 24: 6618.

[82] Nakanishi H, Jean YC. Positronium Formation at Free-volume Sites in the Amorphous Regions of Semicrystalline PEEK. J. Polym. Sci. B 1989; 27: 1419.

[83] Sano M, Murakami H, Ichimura K. Positronium in a layered-structure material: Montmorillonite. J. Radioanal. Nucl. Chem. 1999; 239 (2): 325.

[84] Harms S, Rätzke K, Faupel F, Schneider GJ, Willner L, and Richter D. Free Volume of Interphases in Model Nanocomposites Studied by Positron Annihilation Lifetime Spectroscopy. Macromolecules 2010; 43: 10505. 


\section{Supporting Information}

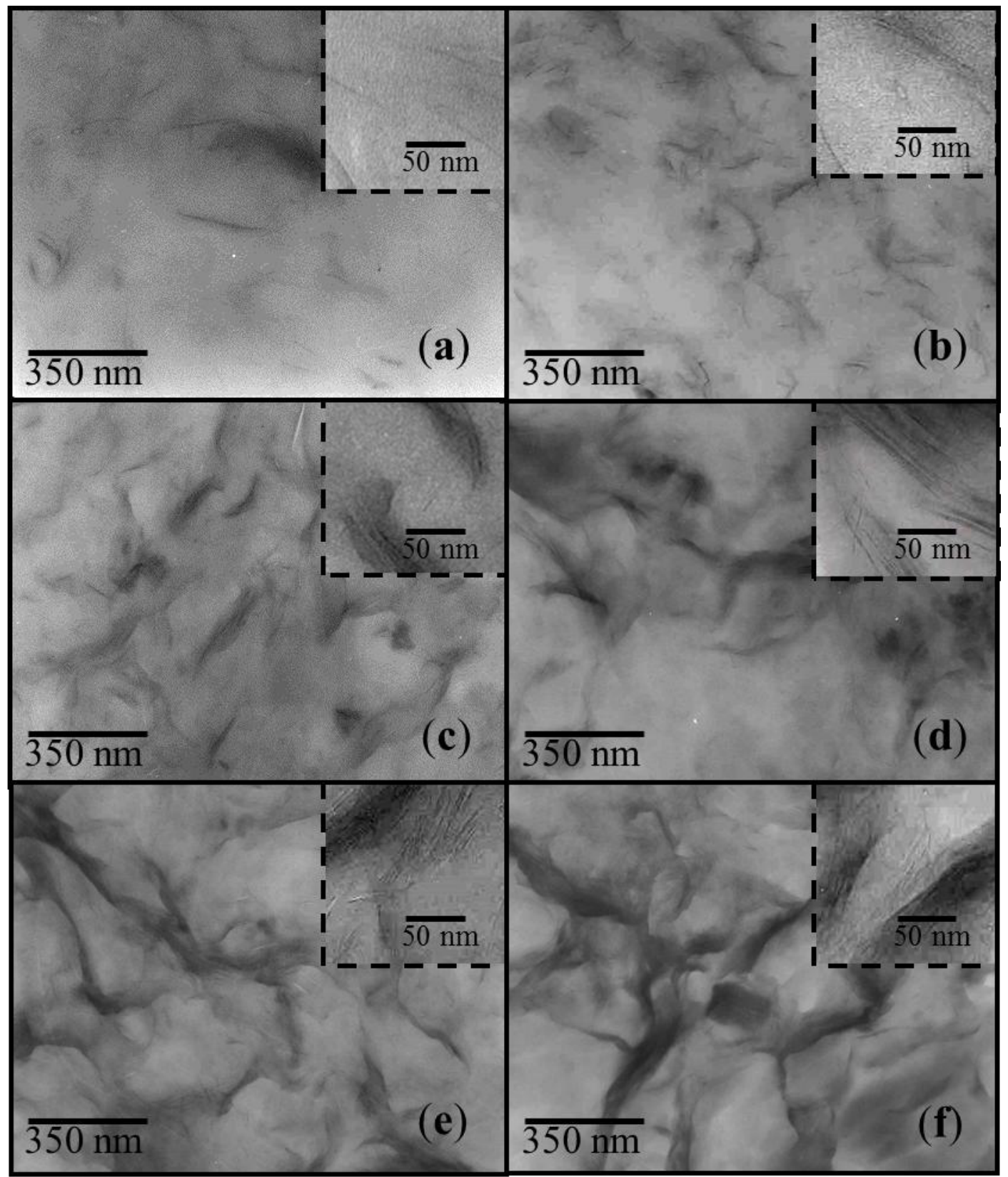

Supporting Fig. 1. Representative high (inserts) and low magnification TEM micrographs of the HBP4/Na ${ }^{+} \mathrm{MMT}$ nanocomposites with (a) 1 , (b) 5 , (c) 15 , (d) 20 , (e) 25 , and (f) $40 \% \mathrm{wt} / \mathrm{wt}$ clay content. 

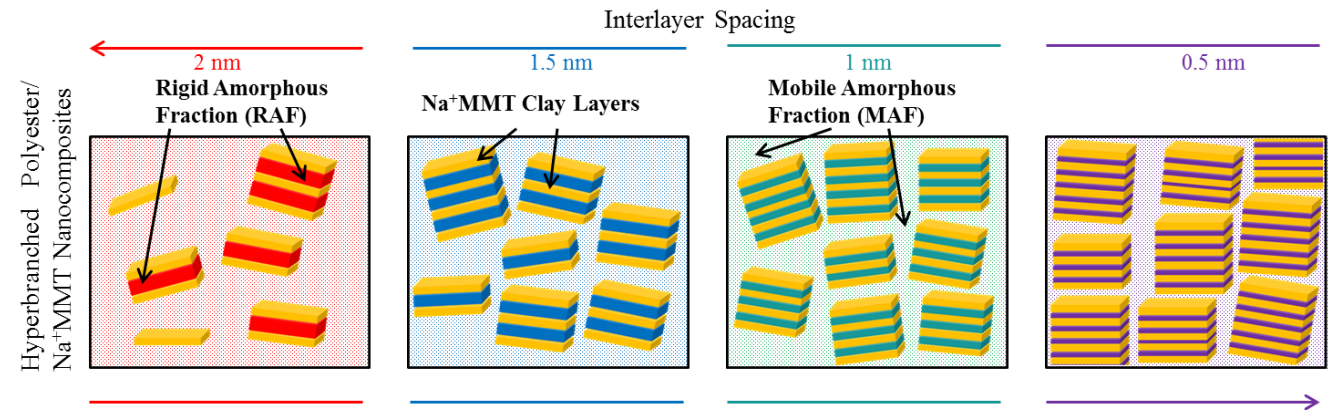

$\mathrm{Na}^{+} \mathrm{MMT}$ Concentration $\left(w_{m}\right)$
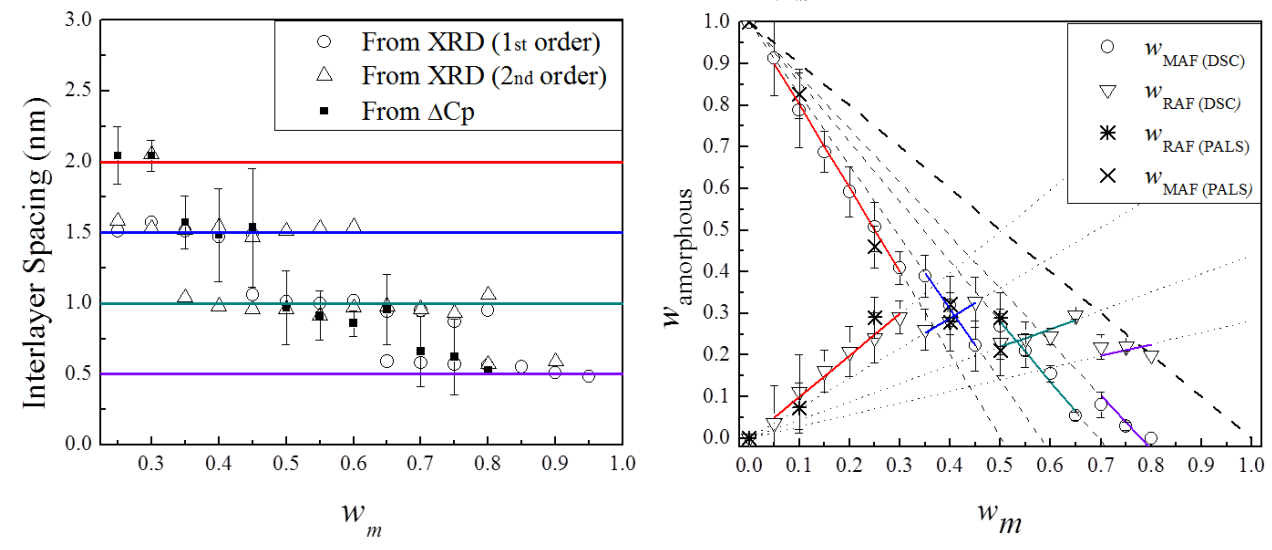